\title{
ISO-SWS calibration and the accurate modelling of cool-star atmospheres ${ }^{\star}$
}

\author{
IV. G9 to M2 stars $\star \star$ \\ L. Decin ${ }^{1, \star \star \star}$, B. Vandenbussche ${ }^{1}$, C. Waelkens ${ }^{1}$, G. Decin ${ }^{1}$, K. Eriksson ${ }^{2}$, B. Gustafsson ${ }^{2}$, \\ B. Plez ${ }^{3}$, and A. J. Sauval ${ }^{4}$ \\ 1 Instituut voor Sterrenkunde, KULeuven, Celestijnenlaan 200B, 3001 Leuven, Belgium \\ 2 Institute for Astronomy and Space Physics, Box 515, 75120 Uppsala, Sweden \\ 3 GRAAL - CC72, Université de Montpellier II, 34095 Montpellier Cedex 5, France \\ ${ }^{4}$ Observatoire Royal de Belgique, Avenue Circulaire 3, 1180 Bruxelles, Belgium
}

Received 22 November 2001 / Accepted 2 December 2002

\begin{abstract}
A detailed spectroscopic study of 11 giants with spectral type from G9 to M2 is presented. The 2.38-4.08 $\mu \mathrm{m}$ wavelength-range of band 1 of ISO-SWS (Short-Wavelength Spectrometers on board of the Infrared Space Observatory) in which many different molecules - with their own dependence on each of the stellar parameters - are absorbing, enables us to estimate the effective temperature, the gravity, the microturbulence, the metallicity, the $\mathrm{CNO}$-abundances, the ${ }^{12} \mathrm{C} /{ }^{13} \mathrm{C}$-ratio and the angular diameter from the ISO-SWS data. Using the Hipparcos' parallax, the radius, luminosity and gravity-inferred mass are derived. The stellar parameters obtained are in good agreement with other published values, though also some discrepancies with values deduced by other authors are noted. For a few stars ( $\delta$ Dra, $\xi$ Dra, $\alpha$ Tuc, H Sco and $\alpha$ Cet) some parameters - e.g. the CNO-abundances - are derived for the first time. By examining the correspondence between different ISO-SWS observations of the same object and between the ISO-SWS data and the corresponding synthetic spectrum, it is shown that the relative accuracy of ISO-SWS in band $1(2.38-4.08 \mu \mathrm{m})$ is better than $2 \%$ for these high-flux sources. The high level of correspondence between observations and theoretical predictions, together with a confrontation of the estimated $T_{\text {eff }}$ (ISO) value with $T_{\text {eff }}$ values derived from colours - which demonstrates the consistency between $V-K, B C_{K}, T_{\text {eff }}$ and $\theta_{\mathrm{d}}$ derived from optical or IR data - proves that both the used MARCS models to derive the stellar quantities and the flux calibration of the ISO-SWS detectors have reached a high level of reliability.
\end{abstract}

Key words. infrared: stars - stars: atmospheres - stars: late-type - stars: fundamental parameters

\section{Introduction}

In the fourth article of this series, we discuss the ISO-SWS (Short-Wavelength Spectrometers on board of the Infrared Space Observatory) spectra of 11 giants cooler than the Sun. These giants are part of a larger sample selected as stellar standard candles for the calibration of the detectors of ISO-SWS. In Decin et al. (2000, hereafter referred to as Paper I) a method was developed to analyse the infrared data $(2.38-12 \mu \mathrm{m})$ of cool stars: the specific dependence of the absorption pattern by the different molecules gives the possibility to estimate fundamental stellar parameters like the effective temperature $\left(T_{\text {eff }}\right)$,

Send offprint requests to: L. Decin,

e-mail: Leen. Decin@ster. kuleuven.ac.be

* Based on observations with ISO, an ESA project with instruments funded by ESA Member States (especially the PI countries France, Germany, The Netherlands and the UK) and with the participation of ISAS and NASA.

$\star \star$ Appendices A-D are only available in electronic form at http://www . edpsciences.org

$\star \star \star$ Postdoctoral Fellow of the Fund for Scientific Research, Flanders. the gravity $(\log g)$, the microturbulence $\left(\xi_{\mathrm{t}}\right)$, the metallicity, the $\mathrm{CNO}$-abundances, the ${ }^{12} \mathrm{C} /{ }^{13} \mathrm{C}$-ratio and the angular diameter $\left(\theta_{\mathrm{d}}\right)$. We now apply this method of analysis to the ISOSWS data of these 11 giants. Keeping the general calibration problems and the accuracy of the theoretical models and synthetic spectra (based on the MARCS and TURBOSPECTRUM code, Gustafsson et al. 1975; Plez et al. 1992; 1993; version May 1998) in mind - as discussed in Decin et al. (2003, hereafter referred to as Paper II) - the error bars on the different stellar parameters are determined and the resultant stellar parameters are confronted with other published parameters.

This paper is organised in the following way: in Sect. 2 a summary of the general discrepancies between the ISO-SWS spectra of the cool giants and the theoretical spectra, as described in Paper II, is given. We will go more deeply into the determination of the different stellar parameters and their error bar in Sect. 3. After an overview of the deduced stellar parameters, each star is discussed in more detail. Not only are the accuracy and precision of the ISO-SWS data assessed, but the deduced stellar parameters are also evaluated with respect to 
other published values. A discussion on some of the stellar parameters obtained is held in Sect. 4 , where we focus on (i) the effective temperature and (ii) the ${ }^{12} \mathrm{C} /{ }^{13} \mathrm{C}$ ratio. The conclusions are given in Sect. 5.

The appendix of this article is published electronically. Most of the grey-scale plots in the printed version of the article are printed in colour in the appendix, in order to better distinguish the different spectra or symbols.

\section{Summary of general discrepancies (Paper II)}

The origin(s) of different types of discrepancies seen in the spectra of the cool stars could be settled from a detailed comparison between ISO-SWS data and theoretical predictions. As far as the calibration is concerned, we demonstrated in Paper II that problems with the relative spectral response functions in band $1 \mathrm{~A}(2.38-2.60 \mu \mathrm{m})$ and band $2(4.08-12 \mu \mathrm{m})$, fringes at the end of band 1D and memory-effects in band 2 destroy the flux accuracy in this wavelength range. Problems with the $\mathrm{CO}$ lines underline that one needs to know the instrumental profile and resolution accurately. Concerning the modelling part, the main problems were situated in the incomplete knowledge of the atomic oscillator strengths in the infrared. The fact that the low-excitation first-overtone $\mathrm{CO}$ lines were predicted as being too strong and the fundamental $\mathrm{OH}$ lines as being to weak, indicated that some of the assumptions the models are based on e.g. hydrostatic equilibrium, homogeneity - are questionable for these cool stars.

\section{Stellar parameters}

As was demonstrated in Paper I, it is possible to estimate different stellar parameters for cool giants from their ISO-SWS spectrum and this due to the presence of many molecular absorbers in the $2.38-4.08 \mu \mathrm{m}$ wavelength range. E.g. the slope of the continuum between $3.1 \mu \mathrm{m}$ and $4.0 \mu \mathrm{m}$ is indicative for the effective temperature $\left(T_{\text {eff }}\right)$, while the slope in band $1 \mathrm{~B}$ and band 1D makes it possible to pin down the gravity (log $g$ ). The microturbulent velocity $\left(\xi_{\mathrm{t}}\right)$, the abundance of carbon, nitrogen and oxygen and the ${ }^{12} \mathrm{C} /{ }^{13} \mathrm{C}$-ratio can be deduced from the relative strength of the molecular features. For the determination of the metallicity $([\mathrm{Fe} / \mathrm{H}])$, both the slope of the continuum and the strength of the features are required (see Fig. 2 in Paper I). Finally, the angular diameter $\left(\theta_{\mathrm{d}}\right)$ can be extracted directly from the absolute flux-level of the ISO-SWS data.

Although, these IR ISO-SWS data give us a lot of information concerning the stellar parameters, an inspection of a large grid with stellar parameters typical for these stars, learned us that there exists an actual danger to be trapped in a local minimum in this huge parameter space. Moreover, the preliminary results of a statistical study (Shkedy et al., in prep.; Decin et al., in prep.) indicate that the maximum error bar on the logarithm of the gravity (as determined from the ISOSWS spectra) can be quite large $(\gtrsim 0.40 \mathrm{dex})$ inducing significant error bars on e.g. the stellar abundances. Hence, we decided to determine first the effective temperature from $(V-K)$ colours (see Sect. 3.1). A first guess for the gravity was found from $K$-band photometry, the bolometric correction $B C_{K}$, the
Hipparcos' parallax $\pi, T_{\text {eff }}(V-K)$ and a mass $M_{\mathrm{hr}}$ estimated from evolutionary tracks (see Sect. 3.1). Using then the ISOSWS spectra, the values of these two parameters were refined if necessary. In this way, the maximum $^{1}$ error on $T_{\text {eff }}$, $\log g, \xi_{\mathrm{t}},[\mathrm{Fe} / \mathrm{H}], \varepsilon(\mathrm{C}), \varepsilon(\mathrm{N}), \varepsilon(\mathrm{O})$ and ${ }^{12} \mathrm{C} /{ }^{13} \mathrm{C}$-ratio could be restricted to $200 \mathrm{~K}, 0.20 \mathrm{dex}, 1 \mathrm{~km} \mathrm{~s}^{-1}, 0.30 \mathrm{dex}, 0.30 \mathrm{dex}$, 0.40 dex, 0.30 dex and 3 respectively. A mean error is then $\sim 2 / 3$ of this maximum error, depending on the quality of the observation. As will be discussed in Sect. 3.13, it was impossible to deduce $T_{\text {eff }}, \log g, \xi_{\mathrm{t}},[\mathrm{Fe} / \mathrm{H}]$ and the CNO-abundances from the ISO-SWS spectrum of $\beta$ Peg. The error bars are estimated from a comparison with other quoted literature values.

\subsection{First guess for $T_{\text {eff }}$ and $\log g$}

One can calculate $T_{\text {eff }}$ directly from $V-K$ using (semi)empirical $\left(T_{\text {eff }}, V-K\right)$ temperature scales. Such a calibration is e.g. given by Bessell et al. (1998), who determined a polynomial fit between $T_{\text {eff }}$ and $V-K$ based on interferometer and IRFM data (Infrared Flux Method Blackwell \& Shallis 1977) for giants. In Table 1 , the $V$ and $K$ magnitude of Simbad are listed. For $\beta \mathrm{UMi}$, we list the $K$ magnitude of Faucherre et al. (1983); for $\alpha$ Tuc and H Sco the $K$ magnitudes found in the atlas of Gezari et al. (1999) are given. The $(V-K)$ colour has been corrected for interstellar extinction using $A_{V}=0.8 \mathrm{mag} / \mathrm{kpc}$ (Blackwell et al. 1990) and $E(V-K)=A_{V} / 1.1$ (Mathis 1990), with the distance calculated from the Hipparcos' parallax (see Table 3). The values for the effective temperature calculated from formula abcd given in Table 7 in Bessell et al. (1998) are listed in Table 1. A comparison with other $\left(T_{\text {eff }}, V-K\right)$ temperature scales will be given in Sect. 4.1. A typical error bar on the derived $T_{\text {eff }}\left((V-K)_{0}\right)$ values is $50 \mathrm{~K}$.

In order to determine the gravity, we need the radius $R$ and the mass. The first parameter is assessed from $K, B C_{K}$, $\pi$ (from Hipparcos) and $T_{\text {eff }}\left((V-K)_{0}\right)$. We used $B C_{K}((V-$ $\left.K)_{0}\right)=m_{\text {bol }}-K$ from Bessell et al. (1998). The work of Bessell et al. (1998) is based on current MARcs-models and synthetic colours, and ensures consistency between derived parameters and model spectra. The error on the bolometric correction is assumed to be $0.05 ; M_{\text {bol. } \odot}$ is assumed to be 4.74 (Bessell et al. 1998). The radii derived in this way are tabulated in Table 2.

Mass values for the stars in our sample are estimated from evolutionary tracks with appropriate metallicity as calculated by Girardi et al. (2000), with the only exception being the more massive star $\alpha$ Car for which the evolutionary tracks of Lejeune \& Schaerer (2001) have been used. The two sets of evolutionary tracks from the zero age main sequence (ZAMS) to the red giant branch (RGB) agree well with each other. Only Girardi et al. (2000) have given the evolutionary tracks from the zero age horizontal branch (ZAHB)

\footnotetext{
1 This maximum error is not the error as obtained from varying one parameter at a time, since this only gives us an underestimate of the uncertainties. An error in one parameter may couple to the error in another parameter "constructively". The effect of this coupling will be different for different type of stars. Considering here the G9 to M2 giants in our sample, a large grid with typical stellar parameters have been inspected in order to estimate this coupling and to determine the maximum error.
} 
Table 1. Magnitude measurements for the Johnson $V$ and $K$ pass-bands are tabulated. The effective temperature (in $\mathrm{K})$ derived from the $\left(T_{\mathrm{eff}}\right.$, $\left.(V-K)_{0}\right)$ relationship as given by Bessell et al. (1998) is listed in the last column.

\begin{tabular}{llrrrrc}
\hline \hline name & HD-number & $V$ & $K$ & $V-K$ & $(V-K)_{0}$ & $T_{\text {eff }}\left((V-K)_{0}\right)$ \\
\hline$\delta$ Dra & HD 180711 & $3.08^{a}$ & $0.80^{b}$ & 2.28 & 2.26 & 4828 \\
$\xi$ Dra & HD 163588 & $3.75^{a}$ & $1.03^{b}$ & 2.72 & 2.70 & 4457 \\
$\alpha$ Boo & HD 124897 & $-0.06^{a}$ & $-2.95^{c}$ & 2.89 & 2.88 & 4331 \\
$\alpha$ Tuc & HD 211416 & $2.86^{g}$ & $-0.09^{f}$ & 2.95 & 2.91 & 4312 \\
$\beta$ UMi & HD 131873 & $2.06^{a}$ & $-1.22^{d}$ & 3.28 & 3.25 & 4111 \\
$\gamma$ Dra & HD 164058 & $2.23^{a}$ & $-1.34^{b}$ & 3.57 & 3.54 & 3970 \\
$\alpha$ Tau & HD 29139 & $0.87^{a}$ & $-2.81^{b, c}$ & 3.68 & 3.67 & 3915 \\
H Sco & HD 149447 & $4.16^{b}$ & $0.00^{h}$ & 4.16 & 4.08 & 3764 \\
$\beta$ And & HD 6860 & $2.03^{a}$ & $-1.83^{c}$ & 3.86 & 3.82 & 3856 \\
$\alpha$ Cet & HD 18884 & $2.49^{a}$ & $-1.63^{e}$ & 4.12 & 4.07 & 3768 \\
$\beta$ Peg & HD 217906 & $2.45^{a}$ & $-2.20^{c}$ & 4.65 & 4.61 & 3615 \\
\hline
\end{tabular}

${ }^{a}$ Mermilliod (1986); ${ }^{b}$ Johnson et al. (1966); ${ }^{c}$ Lee (1970); ${ }^{d}$ Faucherre et al. (1983); ${ }^{e}$ Glass (1974); ${ }^{f}$ Wamsteker (1981); ${ }^{g}$ Nicolet $(1978) ;{ }^{h}$ Price (1968).

to the thermally pulsing asymptotic giant branch (TP-AGB). By plotting $\left(L\left(M_{\text {bol }}\right) \pm \sigma\left(L\left(M_{\text {bol }}\right)\right), T_{\text {eff }}\left((V-K)_{0}\right) \pm \sigma\left(T_{\text {eff }}((V-\right.\right.$ $\left.\left.K)_{0}\right)\right)$ ) for each star on the HR-diagram the mass values $M_{\mathrm{hr}}$ are obtained (see Table 2). Note that most of the giants lie above the horizontal branch and hence could burn hydrogen in a shell surrounding the helium core or could be post-He-flash stars. From our observations no diagnostic tools exist to decide upon the evolutionary status, with the only exception being $\beta$ Peg, since its colours do not match any RGB evolutionary tracks. In Table 2 the mass values are given for a giant on the RGB (except for $\beta$ Peg), with a typical error bar of $0.3 M_{\odot}$ when $M_{\mathrm{hr}} \leq 2.5 M_{\odot}$. When a star would be on the AGB, this value should be lowered by $\sim 0.2 M_{\odot}$.

These radius- and mass values were then used to find firstguess values for the gravity (last column in Table 2). Also the mean error for all these parameters is listed.

\subsection{Results}

Using the ISO-SWS spectra, these first guesses were further refined and the $[\mathrm{Fe} / \mathrm{H}], \varepsilon(\mathrm{C}, \mathrm{N}, \mathrm{O}),{ }^{12} \mathrm{C} /{ }^{13} \mathrm{C}$-ratio and $\theta_{\mathrm{d}}$ were derived. The results from the detailed spectroscopic study and the final error bars are presented in Table 3. The distance $D$ (in pc) is calculated from the parallax measurements (in mas) of Hipparcos. $R\left(D, \theta_{\mathrm{d}}\right)$ in combination with the gravity then yielded the gravity-inferred mass $M_{\mathrm{g}}\left(\right.$ in $\left.M_{\odot}\right)$. The luminosity $L$ (in $\left.L_{\odot}\right)$, extracted from the radius $R\left(D, \theta_{\mathrm{d}}\right)$ and $T_{\text {eff }}$ (ISO), is the last quantity listed in this table. The objects have been sorted by spectral type, but since the spectral type does not necessarily provide a good indicator of the temperature (Tsuji 1981), this ordering is not always by decreasing effective temperature. The theoretical models and synthetic spectra for stars with spectral type earlier than $\mathrm{K} 0$ were calculated using a plane-parallel geometry, for the other ones a spherically symmetric geometry was assumed (see Fig. 1 in Decin et al. 1997). In order to judge upon the goodness-of-fit of the observed and theoretical data, the deviation estimating parameter $\beta$ derived from the Kolmogorov-Smirnov test (see Paper I) has been calculated (see Table 4). This parameter $\beta$ checks the global accordance between the two spectra. The lower the $\beta$-value, the better the accordance. The maximum acceptable $\beta$-values were derived from $\alpha$ Tau in (Paper I). These $\beta_{\max }$ values do not depend on the flux level of the observed target, since the $\beta$ parameter is a global goodness-of-fit parameter and these $\beta_{\max }$ values may thus be used for all observations.

In the following subsections, each of the 11 cool giants will be discussed individually. For each star, calibration details can be found in the appendix. These, in conjunction with the general calibration specifications discussed in Paper II, gives us an idea on the calibration accuracy. If other AOT01 observations ${ }^{2}$ are available, they are compared with each other in the appendix in order to assess the calibration precision of ISO-SWS. Knowing the accuracy and the precision of the observational data, several stellar parameters (and their error bar) are determined from the AOT01 observation and the agreement between observational and theoretical spectra is discussed. Furthermore, the deduced stellar parameters are evaluated with respect to published stellar parameters. Tables with a summary of the different published results, together with a short description of the used methods and/or data and of the assumed and deduced parameters by the different authors quoted in next sections, can be found in the appendix.

\section{3. $\delta$ Dra: AOT01, speed 4, revolution 206 \\ 3.3.1. Comparison between the ISO-SWS spectrum and the synthetic spectrum (Fig. 1)}

Some of the atomic features identified in Fig. 3 in Paper II can still be recognised in $\delta$ Dra, e.g. around $2.55 \mu \mathrm{m}, 2.63 \mu \mathrm{m}$, $3.3 \mu \mathrm{m}, 3.4 \mu \mathrm{m}$ and $3.85 \mu \mathrm{m}$. The second most abundant molecule in nearly all cool stars is $\mathrm{CO}$, because of its very high dissociation energy $(11.09 \mathrm{eV})$, and because of the high

\footnotetext{
${ }^{2}$ Each observation is determined uniquely by its observation number ( 8 digits), in which the first three digits represent the revolution number. The observing data can be calculated from the revolution number which is the number of days after 17 November 1995.
} 
Table 2. Magnitude measurements for the Johnson $K$ band are tabulated. The absolute bolometric magnitude $M_{\text {bol }}$ and the luminosity $L\left(M_{\mathrm{bol}}\right)$ (in $\left.L_{\odot}\right)$ are derived from the $(V-K)_{0}$ colour index, the bolometric correction $B C_{K}\left((V-K)_{0}\right)$ and the Hipparcos' parallax $\pi$. The radius $R$ is calculated from $L\left(M_{\mathrm{bol}}\right)$ and $T_{\mathrm{eff}}\left((V-K)_{0}\right)$. Mass values estimated from evolutionary tracks are given in $M_{\odot}$ for all stars in our sample. A typical error bar for $M_{\mathrm{hr}}$ is $0.3 M_{\odot}$ when $M_{\mathrm{hr}} \leq 2.5 M_{\odot}$. The gravity is derived from $R$ and $M_{\mathrm{hr}}$. More details are given in the text.

\begin{tabular}{lrrrrcccc}
\hline \hline name & \multicolumn{1}{c}{$K_{0}$} & \multicolumn{1}{c}{$B C_{K}$} & \multicolumn{1}{c}{$m_{\mathrm{bol}}$} & $M_{\mathrm{bol}}\left(m_{\mathrm{bol}}, \pi\right)$ & $L\left(M_{\mathrm{bol}}\right)$ & $R\left(L\left(M_{\mathrm{bol}}\right), T_{\mathrm{eff}}\left((V-K)_{0}\right)\right)$ & $M_{\mathrm{hr}}$ & $\log g$ \\
\hline$\delta$ Dra & 0.80 & 1.94 & $2.74 \pm 0.05$ & $0.30 \pm 0.06$ & $59 \pm 3$ & $11.01 \pm 0.37$ & 2.2 & $2.70 \pm 0.07$ \\
$\xi$ Dra & 1.03 & 2.22 & $3.25 \pm 0.05$ & $0.58 \pm 0.06$ & $46 \pm 3$ & $11.40 \pm 0.41$ & 1.1 & $2.36 \pm 0.12$ \\
$\alpha$ Boo & -2.95 & 2.31 & $-0.64 \pm 0.05$ & $-0.90 \pm 0.05$ & $180 \pm 9$ & $23.81 \pm 0.80$ & 1.1 & $1.72 \pm 0.12$ \\
$\alpha$ Tuc & -0.10 & 2.33 & $2.23 \pm 0.05$ & $-1.70 \pm 0.05$ & $376 \pm 32$ & $34.72 \pm 1.69$ & 2.5 & $1.75 \pm 0.07$ \\
$\beta$ UMi & -1.22 & 2.46 & $1.24 \pm 0.05$ & $-1.71 \pm 0.07$ & $379 \pm 23$ & $38.36 \pm 1.50$ & 2.2 & $1.61 \pm 0.07$ \\
$\gamma$ Dra & -1.34 & 2.55 & $1.21 \pm 0.05$ & $-2.07 \pm 0.07$ & $530 \pm 33$ & $48.61 \pm 1.94$ & 1.80 & $1.31 \pm 0.08$ \\
$\alpha$ Tau & -2.81 & 2.59 & $-0.22 \pm 0.05$ & $-1.72 \pm 0.06$ & $395 \pm 23$ & $42.62 \pm 1.67$ & 1.2 & $1.26 \pm 0.11$ \\
H Sco & -0.01 & 2.70 & $2.69 \pm 0.05$ & $-2.40 \pm 0.19$ & $716 \pm 122$ & $62.87 \pm 5.61$ & 1.4 & $0.99 \pm 0.12$ \\
$\beta$ And & -1.84 & 2.63 & $0.79 \pm 0.05$ & $-3.14 \pm 0.11$ & $1415 \pm 147$ & $84.23 \pm 4.88$ & 2.2 & $0.93 \pm 0.08$ \\
$\alpha$ Cet & -1.64 & 2.70 & $1.06 \pm 0.05$ & $-3.09 \pm 0.13$ & $1356 \pm 164$ & $86.35 \pm 5.71$ & 2.5 & $0.96 \pm 0.08$ \\
$\beta$ Peg & -2.21 & 2.80 & $0.59 \pm 0.05$ & $-3.34 \pm 0.11$ & $1702 \pm 169$ & $105.10 \pm 5.97$ & 1.85 & $0.66 \pm 0.09$ \\
\hline
\end{tabular}

${ }^{a}$ Johnson et al. (1966); ${ }^{b}$ Lee (1970); ${ }^{c}$ Faucherre et al. (1983); ${ }^{d}$ Glass (1974).

cosmic abundance of carbon and oxygen. From $\delta$ Dra on, the first-overtone $\mathrm{CO}$ band dominates the spectrum till $2.8 \mu \mathrm{m}$. The problems with these $\mathrm{CO}$ lines are described in point 2 in Paper II. In band $1 \mathrm{~B}, 1 \mathrm{D}$ and $1 \mathrm{E}$ the $\mathrm{OH} \Delta v=1$ lines become visible. Problems with memory effects (Paper II) make it impossible to improve the data in band 2 .

The carbon abundance is deduced from the CO lines in band 1A (where the standard deviation is larger than for the other sub-bands in band 1), the $\mathrm{N}$-abundance from the $\mathrm{CN}$ lines which are almost invisible, and the $\mathrm{O}$-abundance from $\mathrm{OH}$ lines which are also still very weak; the overall uncertainty on the abundance of carbon, nitrogen and oxygen is estimated to be 0.25 dex. Since the chemical equilibrium determines the shape of the synthetic spectrum, too inaccurate a nitrogen abundance would result in high $\beta$-values. So, although the $\mathrm{CN}$ and $\mathrm{NH}$ lines remain also very weak for the cooler giants, a wrong nitrogen abundance can be traced.

\subsubsection{Comparison with other published stellar parameters (see Table D.1 in the appendix)}

The temperatures given by the different authors quoted in Table D.1 all agree quite well. Only the temperature given by Faucherre et al. (1983) and Gustafsson et al. (1974) are somewhat lower than the other values. Gustafsson et al. (1974) have established the temperature scale with the aid of a new theoretical calibration of $R-I$. This colour was computed from scaled solar model atmospheres, with the line blocking considered in a statistical way. The use of scaled solar model atmospheres led to underestimates of $T_{\text {eff }}$, an effect which is also noticeable for the $T_{\text {eff-value of } \xi \text { Dra in Table D.2. }}$

There is a rather large difference in published gravities, being either around 2.9 dex or around 2.1 dex. Only Gratton et al. (1982) and Gratton (1983) have tabulated a value of $\log g=2.1$, a result which was obtained from a curve of growth technique. Also the gravity value given by Burnashev (1983) - who has used narrow-band photometric colours in
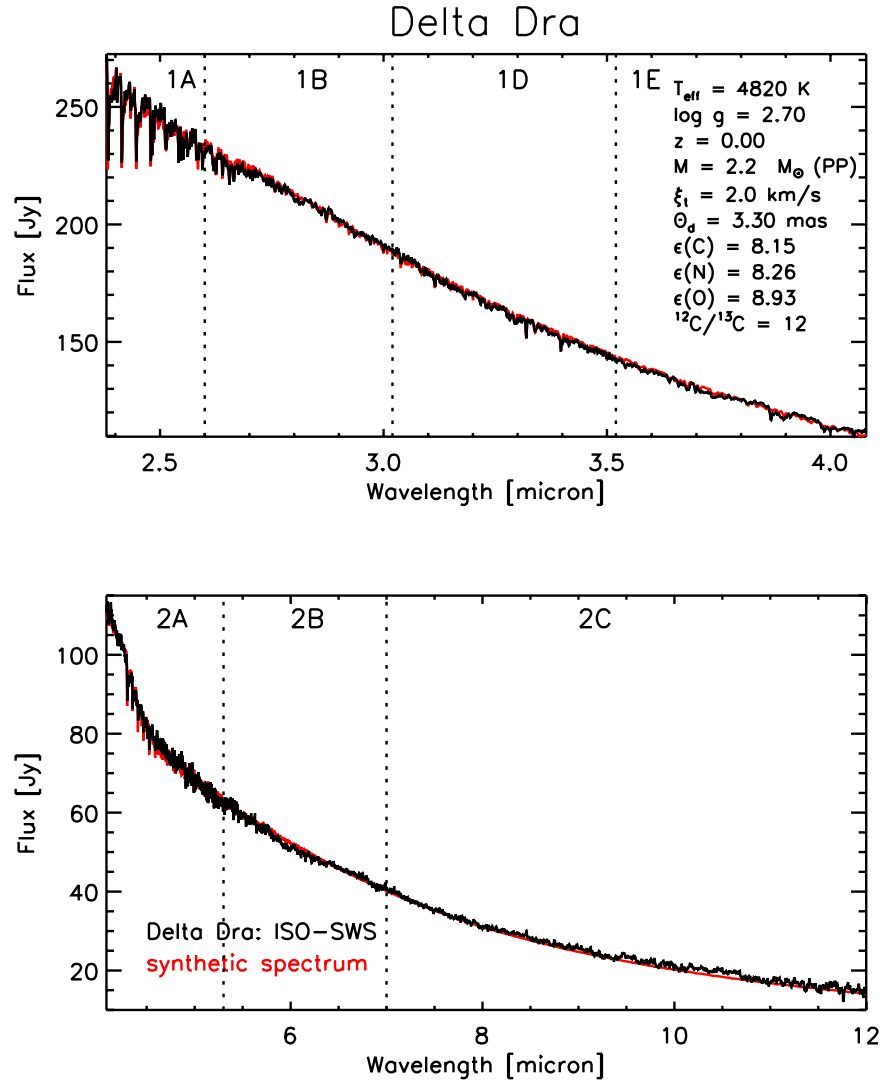

Fig. 1. Comparison between band 1 and band 2 of the ISO-SWS data of $\delta$ Dra (black) and the synthetic spectrum (grey) with stellar parameters $T_{\text {eff }}=4820 \mathrm{~K}, \log g=2.70, M=2.2 M_{\odot},[\mathrm{Fe} / \mathrm{H}]=$ $0.00, \xi_{\mathrm{t}}=2.0 \mathrm{~km} \mathrm{~s}^{-1},{ }^{12} \mathrm{C} /{ }^{13} \mathrm{C}=12, \varepsilon(\mathrm{C})=8.15, \varepsilon(\mathrm{N})=8.26, \varepsilon(\mathrm{O})=$ 8.93 and $\theta_{\mathrm{d}}=3.30$ mas. A coloured version of this plot is available in the appendix as Fig. B.1.

the visible part of the electromagnetic spectrum - is quite discrepant from the more common listed value of $\log g=$ 2.9. We note that also for other stars in our sample, quite some gravity values do not correspond with the ones given by 
Table 3. Final fundamental stellar parameters for the selected stars in the sample. The effective temperature $T_{\text {eff }}$ is given in $K$, the logarithm of the gravity in cgs units, the microturbulent velocity $\xi_{\mathrm{t}}$ in $\mathrm{km} \mathrm{s}^{-1}$, the angular diameter in mas, the parallax $\pi$ in mas, the distance $D$ in parsec, the radius $R\left(D, \theta_{\mathrm{d}}\right)$ in $R_{\odot}$, the gravity-inferred mass $M_{\mathrm{g}}$ in $M_{\odot}$ and the luminosity $L$ in $L_{\odot}$.

\begin{tabular}{|c|c|c|c|c|c|c|c|c|c|c|c|}
\hline & \multicolumn{2}{|c|}{$\delta$ Dra } & \multicolumn{2}{|c|}{$\xi$ Dra } & \multicolumn{2}{|c|}{$\alpha$ Boo } & \multicolumn{2}{|c|}{$\alpha$ Tuc } & \multicolumn{2}{|c|}{$\beta \mathrm{UMi}$} & $\gamma$ Dra \\
\hline Sp. Type & \multicolumn{2}{|c|}{ G9 III } & \multicolumn{2}{|c|}{ K2 III } & \multicolumn{2}{|c|}{ K2 IIIp } & \multicolumn{2}{|c|}{ K3 III } & \multicolumn{2}{|c|}{ K4 III } & K5 III \\
\hline \multirow{2}{*}{$\begin{array}{l}T_{\text {eff }} \\
\log g\end{array}$} & \multicolumn{2}{|c|}{$4820 \pm 140$} & \multicolumn{2}{|c|}{$4440 \pm 300$} & \multicolumn{2}{|c|}{$4320 \pm 140$} & \multicolumn{2}{|c|}{$4300 \pm 140$} & \multicolumn{2}{|c|}{$4085 \pm 140$} & $3960 \pm 140$ \\
\hline & \multicolumn{2}{|c|}{$2.70 \pm 0.20$} & \multicolumn{2}{|c|}{$2.40 \pm 0.25$} & \multicolumn{2}{|c|}{$1.50 \pm 0.15$} & \multicolumn{2}{|c|}{$1.35 \pm 0.15$} & \multicolumn{2}{|c|}{$1.60 \pm 0.15$} & $1.30 \pm 0.25$ \\
\hline$\xi_{\mathrm{t}}$ & \multicolumn{2}{|c|}{$2.0 \pm 0.5$} & \multicolumn{2}{|c|}{$2.0 \pm 1.0$} & \multicolumn{2}{|c|}{$1.7 \pm 0.5$} & \multicolumn{2}{|c|}{$1.7 \pm 0.5$} & $2.0 \pm$ & 0.5 & $2.0 \pm 0.5$ \\
\hline$[\mathrm{Fe} / \mathrm{H}]$ & 0.00 & 0.25 & 0.10 & 0.30 & -0.50 & 0.20 & $0.00 \pm$ & 0.20 & -0.15 & 0.20 & $0.00 \pm 0.20$ \\
\hline$\varepsilon(\mathrm{C})$ & 8.15 & 0.25 & 8.00 & 0.30 & $7.96 \pm$ & 0.20 & $8.30 \pm$ & 0.20 & $8.25 \pm$ & 0.20 & $8.15 \pm 0.25$ \\
\hline$\varepsilon(\mathrm{N})$ & 8.26 & 0.25 & 8.26 & 0.40 & $7.61 \pm$ & 0.25 & $8.26 \pm$ & 0.25 & $8.16 \pm$ & 0.25 & $8.26 \pm 0.25$ \\
\hline$\varepsilon(\mathrm{O})$ & 8.93 & 0.25 & 8.93 & 0.30 & $8.68 \pm$ & 0.20 & $8.93=$ & 0.20 & $8.83 \pm$ & 0.20 & $8.93 \pm 0.20$ \\
\hline${ }^{12} \mathrm{C} /{ }^{13} \mathrm{C}$ & & \pm 3 & & & $7 \pm$ & & 13 & & $9 \pm$ & & $10 \pm 2$ \\
\hline$\theta_{\mathrm{d}}$ & 3.30 & 0.20 & 3.11 & 0.22 & $20.72=$ & 1.28 & $6.02=$ & 0.37 & $9.93 \pm$ & 0.62 & $9.98 \pm 0.63$ \\
\hline$\pi$ & 32.5 & \pm 0.46 & 29.26 & 0.49 & 88.85 & 0.74 & 16.42 & 0.59 & 25.79 & 0.52 & $22.10 \pm 0.46$ \\
\hline$D$ & 30.73 & \pm 0.43 & 34.34 & $=0.57$ & $11.26=$ & 0.09 & 60.90 & 2.19 & 38.78 & 0.78 & $45.25 \pm 0.94$ \\
\hline$R$ & 10.9 & \pm 0.68 & 11.42 & 0.83 & $25.06=$ & 1.56 & 39.40 & 2.82 & 41.38 & 2.71 & $48.53 \pm 3.23$ \\
\hline$M_{\mathrm{g}}$ & 2.18 & 1.04 & 1.20 & 0.71 & $0.73 \pm$ & 0.27 & $1.27=$ & 0.48 & $2.49 \pm$ & 0.92 & $1.72 \pm 1.02$ \\
\hline$L$ & & 10 & & 14 & $197=$ & 36 & 479 & 93 & 430 & 82 & $523 \pm 101$ \\
\hline & & $\alpha \mathrm{T}$ & & & & & & & & & Peg \\
\hline & Type & K5 & & & & & III & & III & $\mathrm{M} 2$ & 5 III \\
\hline$T$ & & 3850 & 140 & 3900 & $=140$ & 3880 & \pm 140 & 3740 & \pm 140 & 3600 & \pm 300 \\
\hline & & $1.50 \pm$ & 0.15 & 1.30 & 0.15 & 0.95 & $=0.25$ & 0.95 & $=0.25$ & 0.65 & \pm 0.40 \\
\hline$\xi_{\mathrm{t}}$ & & $1.7 \pm$ & 0.5 & 2.0 & 0.5 & 2.0 & 0.5 & 2.3 & $=0.5$ & & \pm 0.3 \\
\hline & & -0.15 & 0.20 & 0.00 & 0.20 & 0.00 & 0.30 & 0.00 & $=0.30$ & & 00 \\
\hline & & $8.35 \pm$ & 0.20 & 8.25 & 0.20 & 8.12 & $=0.30$ & 8.20 & $=0.30$ & 8.20 & \pm 0.40 \\
\hline & & $8.35=$ & 0.25 & 8.26 & 0.25 & 8.37 & $=0.40$ & 8.26 & $=0.40$ & 8.18 & \pm 0.40 \\
\hline & & $8.93=$ & 0.20 & 8.83 & 0.20 & 9.08 & $=0.30$ & 8.93 & $=0.30$ & 8.93 & \pm 0.40 \\
\hline & ${ }^{13} \mathrm{C}$ & 10 & & & & & $=2$ & & \pm 2 & & \pm 3 \\
\hline$\theta_{\mathrm{d}}$ & & 20.77 & 1.31 & 4.73 & 0.30 & 13.30 & \pm 0.84 & 12.52 & \pm 0.79 & 16.60 & \pm 1.32 \\
\hline$\pi$ & & 50.09 & 0.95 & 9.63 & 0.79 & 16.36 & \pm 0.76 & 14.82 & \pm 0.83 & 16.3 & \pm 0.72 \\
\hline$D$ & & 19.96 & 0.38 & 103.84 & \pm 8.52 & 61.12 & \pm 2.84 & 67.48 & \pm 3.78 & 61.08 & \pm 2.69 \\
\hline$R$ & & 44.56 & 2.94 & 52.79 & 5.47 & 87.37 & \pm 6.85 & 90.79 & \pm 7.66 & 108.9 & \pm 9.90 \\
\hline$M$ & & $2.30 \pm$ & 0.85 & 2.03 & 0.82 & 2.49 & $=1.48$ & 2.69 & $=1.61$ & & $\begin{array}{l}+4.27 \\
-1.34\end{array}$ \\
\hline$L$ & & 394 & 77 & 582 & 147 & 1561 & \pm 332 & 1455 & \pm 328 & 180 & \pm 683 \\
\hline
\end{tabular}

Burnashev (1983). Possible reasons include inaccurate spectrophotometric data or inaccurate formulae to convert the colour indices to the stellar parameter values. Using a gravity of $\log g=2.10$, the best fit was obtained with a - low - carbon abundance of $\varepsilon(C)=8.00$ (Fig. 2). The discrepancies are more pronounced than in Fig. 1, while the $\beta$-values are larger than for $\log g=2.70$ and $\varepsilon(C)=8.15$.

Other indirect angular diameter determinations were not found. Gratton et al. (1982) have derived the radius from the visual surface brightness and the temperature, and so have obtained the mass $M_{\mathrm{g}}$ of $\delta$ Dra. Faucherre et al. (1983) measured the angular diameter with the two-telescope stellar interferometer (I2T) at CERGA. By using $D=36 \pm 8$ pc (Jenkins 1952), a radius $R=15 \pm 4 R_{\odot}$ was obtained. Although different methods and/or data are used, the three values for the radius listed in Table D. 1 are in good agreement.

From the $10 \%$ absolute flux level accuracy, the uncertainty on the angular diameter $\left(\theta_{\mathrm{d}}=3.30 \mathrm{mas}\right)$ is estimated to be 0.20 mas. This yields a gravity-inferred mass of $M_{\mathrm{g}}=$ $2.18 \pm 1.04 M_{\odot}$. This value for $M_{\mathrm{g}}$ is higher than the gravityinferred mass deduced by Gratton et al. (1982), due to their use of a lower gravity value.

\section{4. $\xi$ Dra: AOT01, speed 3, revolution 314}

\subsubsection{Comparison between the ISO-SWS spectrum and the synthetic spectrum (Fig. 3)}

The larger pointing offset in the $y$-direction and the high noise level reduce the reliability of the shape of the sub-bands. Especially the shape of the data at the end of band $1 \mathrm{~A}$, in band $1 \mathrm{~B}$ and in band $1 \mathrm{D}$ seems unreliable. As a result, the uncertainties on the fundamental stellar parameters are enhanced. Fortunately, the post-He spectrum does not display these problems. Using then both the speed-3 observation and the post-He data, the stellar parameters were estimated. Similar 
Table 4. The $\beta$-values from the Kolmogorov-Smirnov test are given not only for the different sub-bands, but also for the different molecular absorbers the $\beta$-values. In the second column, one can find the maximum acceptable $\beta$-value for that specific wavelength-range as estimated for a high-quality AOT01 speed-4 observation (Paper I).

\begin{tabular}{lccccccccccccc}
\hline \hline & $\beta_{\max }$ & $\delta$ Dra & $\xi$ Dra & $\alpha$ Boo & $\alpha$ Tuc & $\beta$ UMi & $\gamma$ Dra & $\alpha$ Tau & H Sco & $\beta$ And & $\alpha$ Cet & $\beta$ Peg \\
\hline$\beta_{1 \mathrm{~A}}$ & 0.06 & 0.051 & 0.074 & 0.015 & 0.036 & 0.050 & 0.058 & 0.040 & 0.033 & 0.049 & 0.057 & 0.157 \\
$\beta_{1 \mathrm{~B}}$ & 0.05 & 0.046 & 0.072 & 0.041 & 0.013 & 0.029 & 0.048 & 0.034 & 0.026 & 0.046 & 0.041 & 0.150 \\
$\beta_{1 \mathrm{D}}$ & 0.04 & 0.040 & 0.069 & 0.036 & 0.021 & 0.025 & 0.011 & 0.036 & 0.019 & 0.019 & 0.024 & 0.218 \\
$\beta_{1 \mathrm{E}}$ & 0.04 & 0.051 & 0.022 & 0.043 & 0.035 & 0.040 & 0.014 & 0.027 & 0.033 & 0.044 & 0.044 & 0.084 \\
& & & & & & & & & & & & \\
$\beta_{\mathrm{CO}(1 \mathrm{~A})}$ & 0.06 & 0.051 & 0.074 & 0.015 & 0.036 & 0.050 & 0.058 & 0.040 & 0.033 & 0.040 & 0.057 & 0.157 \\
$\beta_{\mathrm{CO}(1 \mathrm{~B})}$ & 0.05 & 0.029 & 0.044 & 0.038 & 0.017 & 0.017 & 0.026 & 0.039 & 0.034 & 0.056 & 0.038 & 0.167 \\
$\beta_{\mathrm{OH} / \mathrm{H}_{2} \mathrm{O}(1 \mathrm{~B})}$ & 0.04 & 0.010 & 0.010 & 0.010 & 0.027 & 0.020 & 0.023 & 0.024 & 0.021 & 0.032 & 0.019 & 0.027 \\
$\beta_{\mathrm{OH} / \mathrm{H}_{2} \mathrm{O}(1 \mathrm{D})}$ & 0.04 & 0.040 & 0.069 & 0.036 & 0.021 & 0.025 & 0.011 & 0.036 & 0.019 & 0.020 & 0.024 & 0.218 \\
$\beta_{\mathrm{OH}(\mathrm{IE})}$ & 0.03 & 0.038 & 0.019 & 0.041 & 0.023 & 0.042 & 0.017 & 0.025 & 0.017 & 0.031 & 0.036 & 0.047 \\
$\beta_{\mathrm{SiO}(1 \mathrm{E})}$ & 0.03 & 0.024 & 0.015 & 0.014 & 0.021 & 0.028 & 0.021 & 0.022 & 0.013 & 0.018 & 0.018 & 0.027 \\
\hline
\end{tabular}

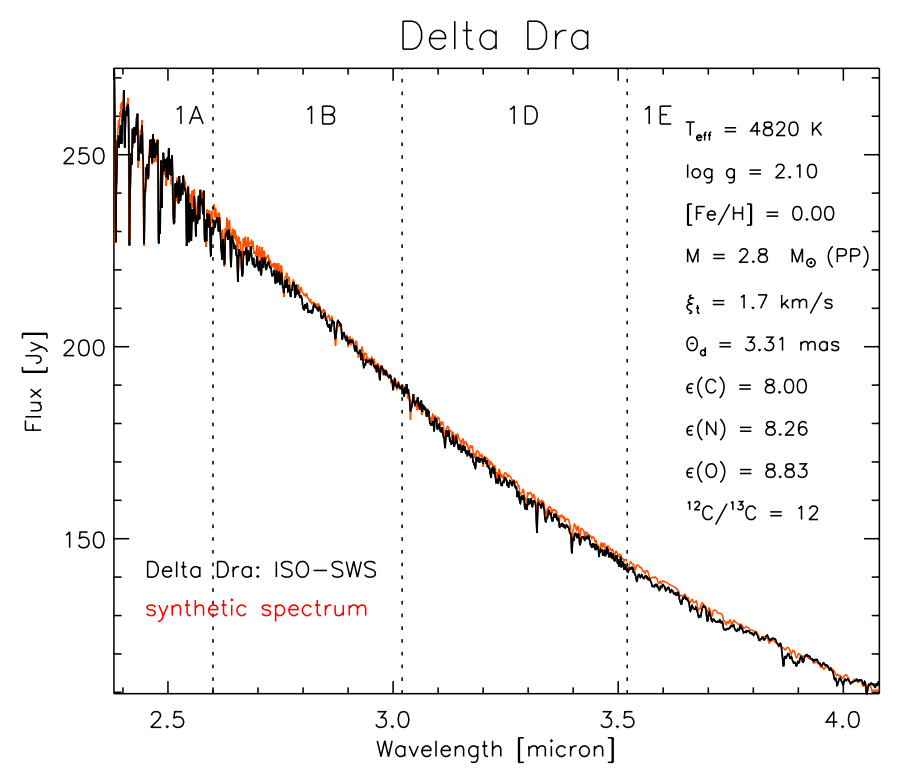

Fig. 2. Comparison between band 1 of the ISO-SWS data (rev. 206) of $\delta$ Dra (black) and the synthetic spectrum (grey) with stellar parameters $T_{\text {eff }}=4820 \mathrm{~K}, \log g=2.10, M=2.8 M_{\odot},[\mathrm{Fe} / \mathrm{H}]=0.00$, $\xi_{\mathrm{t}}=1.7 \mathrm{~km} \mathrm{~s}^{-1},{ }^{12} \mathrm{C} /{ }^{13} \mathrm{C}=12, \varepsilon(\mathrm{C})=8.00, \varepsilon(\mathrm{N})=8.26, \varepsilon(\mathrm{O})=8.83$ and $\theta_{\mathrm{d}}=3.31$ mas. A coloured version of this plot is available in the appendix as Fig. B.2.

discrepancies as seen in Fig. 1 of $\delta$ Dra are also arising in the comparison between the observational data of $\xi$ Dra and its synthetic spectrum. Some examples caused by atomic transitions may be seen around $2.545 \mu \mathrm{m}, 2.595 \mu \mathrm{m}$ and $2.623 \mu \mathrm{m}$. The first-overtone CO lines are clearly visible around $2.4 \mu \mathrm{m}$. As described in Paper II, the strongest CO lines were predicted as somewhat too strong when a resolution of 1500 is used. The carbon, nitrogen and oxygen abundance, together with the ${ }^{12} \mathrm{C} /{ }^{13} \mathrm{C}$ ratio are an indication that $\xi$ Dra has already gone through the first dredge-up (see Sect. 4.2). At $4.005 \mu \mathrm{m}$, the $2-0$ band head of the $\mathrm{SiO}$ first-overtone band emerges.

The post-helium observation is also compared with a synthetic spectrum generated with the same stellar parameters (but other $\theta_{\mathrm{d}}$ due to the higher absolute flux in the post-He observation) as in Fig. 3 at an appropriate resolution (Fig. 4). The corresponding $\beta$-values are $\beta_{1 \mathrm{~A}}=0.089, \beta_{1 \mathrm{~B}}=0.105, \beta_{1 \mathrm{D}}=0.087$ and $\beta_{1 \mathrm{E}}=0.048$. The larger $\beta$-values are due to the very strong Mg-Si-Al-Fe feature around $2.55 \mu \mathrm{m}$ in band 1A (see Fig. 3 in Paper II) and the still ongoing calibration of the post-He data. The spectrum resembles the spectrum of $\delta$ Dra.

\subsubsection{Comparison with other published stellar parameters (see Table D.2 in the appendix)}

As for $\delta$ Dra, the temperature given by Gustafsson et al. (1974) is lower than other published values (see the comment given in Sect. 3.3.2). The different values quoted for the logarithm of the gravity are in close agreement, with the maximum difference being 0.30 dex and our deduced gravity value being close to the mean. The lowest value was found by Brown et al. (1989) by using the $K$-line absolute magnitude $M_{V}(K)$ and the highest value was obtained by McWilliam (1990) by using the wellknown relation between $g, T_{\text {eff }}, L$ and a mass value inferred from evolutionary tracks.

The angular diameter deduced from the speed-3 observation tallies very well with the IRFM angular diameter value given by Blackwell et al. (1991) - which is a revision of Blackwell et al. (1990). Also Gratton et al. (1982) deduced a gravity-inferred mass $M_{\mathrm{g}}$, their value being somewhat higher than ours due to their use of a higher radius value.

For the first time, the abundances of $\mathrm{C}, \mathrm{N}$ and $\mathrm{O}$ are determined. Since the spectroscopic analysis was done on the basis of a speed-3 observation and a post-He speed-4 observation, the error bar on the abundances is estimated to be $\sim 0.30$ dex.

\section{5. $\alpha$ Boo: AOT01, speed 4, revolution 452}

\subsubsection{Comparison between the ISO-SWS spectrum and the synthetic spectrum (Fig. 5)}

The higher standard deviation in band $1 \mathrm{~A}$ and possible uncertainties in the temperature structure in the outer layers determine the maximal acceptable $\beta$-value in band 1A. The 

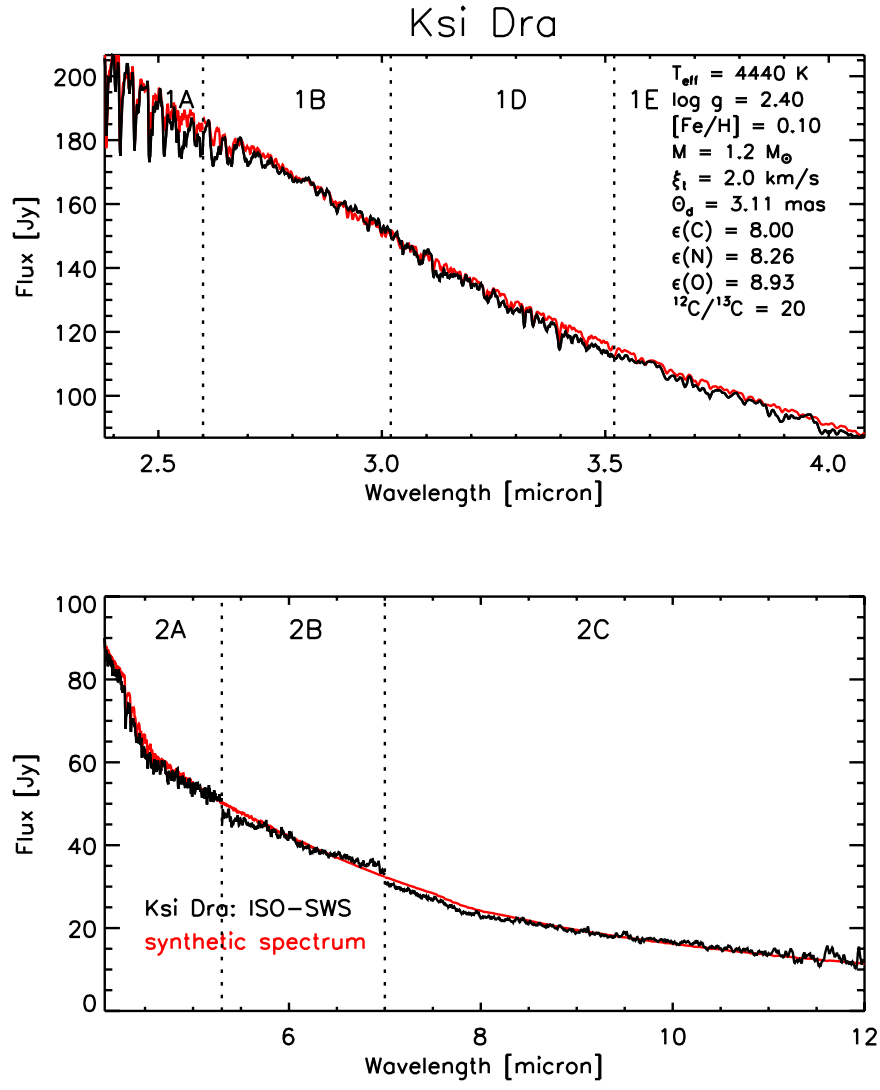

Fig. 3. Comparison between band 1 and band 2 of the ISO-SWS data (rev. 314) of $\xi$ Dra (black) and the synthetic spectrum (grey) with stellar parameters $T_{\text {eff }}=4440 \mathrm{~K}, \log g=2.40, M=1.2 M_{\odot}$, $[\mathrm{Fe} / \mathrm{H}]=0.10, \xi_{\mathrm{t}}=2.0 \mathrm{~km} \mathrm{~s}^{-1},{ }^{12} \mathrm{C} /{ }^{13} \mathrm{C}=20, \varepsilon(\mathrm{C})=8.00, \varepsilon(\mathrm{N})=$ $8.26, \varepsilon(\mathrm{O})=8.93$ and $\theta_{\mathrm{d}}=3.11$ mas. A coloured version of this plot is available in the appendix as Fig. B.3.

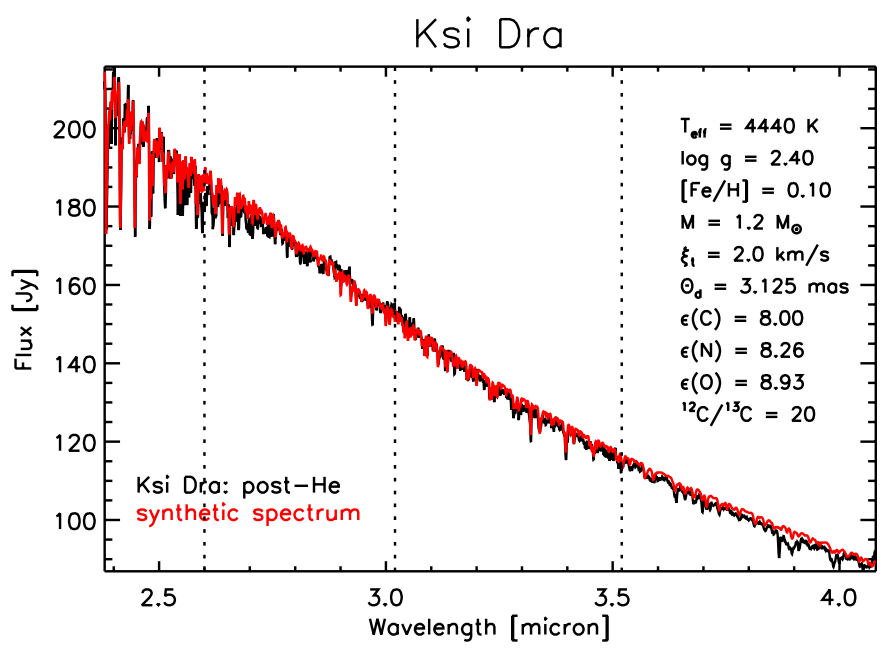

Fig. 4. Comparison between band 1 of the post-helium ISO-SWS data of $\xi$ Dra (black) and the synthetic spectrum (grey) with stellar parameters $T_{\text {eff }}=4440 \mathrm{~K}, \log g=2.40, M=1.2 M_{\odot},[\mathrm{Fe} / \mathrm{H}]=$ $0.10, \xi_{\mathrm{t}}=2.0 \mathrm{~km} \mathrm{~s}^{-1},{ }^{12} \mathrm{C} /{ }^{13} \mathrm{C}=20, \varepsilon(\mathrm{C})=8.00, \varepsilon(\mathrm{N})=8.26, \varepsilon(\mathrm{O})=$ 8.93 and $\theta_{\mathrm{d}}=3.125$ mas. A coloured version of this plot is available in the appendix as Fig. B.4.

$\mathrm{OH}$ lines are predicted as somewhat too weak (Paper II). From $\alpha$ Boo on, the $\mathrm{OH}$ lines determine the spectral signature of band 1D. In band 1E, one can see atomic features around 3.85 $\mu \mathrm{m}$ (see Fig. 3 in Paper II) and the SiO lines around $4 \mu \mathrm{m}$. At the beginning of this project, the first-overtone $\mathrm{SiO}$ lines were always predicted as too strong. At that time, the line list of Tipping \& Chackerian (1981) was used. An extensive theoretical study (Drira et al. 1997) and a comparison with observed SiO lines (Langhoff \& Bauschlicher 1993, Tsuji et al. 1994) revealed that the Einstein coefficients based on the results of Tipping \& Chackerian (1981) are about a factor two larger for the 2-0 overtone band than the currently accepted value. This explained the overestimate of the strength of the $\mathrm{SiO}$ lines. Presently, the more accurate $\mathrm{SiO}$ line list of Langhoff \& Bauschlicher (1993) is used, resulting in a good agreement between observed and calculated spectra. The fundamental bands of $\mathrm{SiO}$ and $\mathrm{CO}$ are visible in band 2, although no quantitative statements can be made due to the problems with the memory effects. The memory effects in band 2 are quite strong due to the high flux level of $\alpha$ Boo in the infrared.

The high-quality data made it possible to pin down the atmospheric parameters (see Paper II). Where the first set of input parameters contained as gravity $\log g=1.75$, the ISO-SWS (and FTS-KP) spectra gave indications that this value could be refined to $\log g=1.50$.

\subsubsection{Comparison with other published stellar parameters (see Table D. 3 in the appendix)}

The different temperature values deduced for $\alpha$ Boo vary around $4300 \mathrm{~K}$, with the lowest value being $4060 \mathrm{~K}$ (Scargle \& Strecker 1979) and the highest value 4628 K (Dyck et al. 1998). A quite often quoted value is the one of Frisk et al. (1982), $T_{\text {eff }}=4375 \mathrm{~K}$, who used photometry in the $0.4-2.2 \mu \mathrm{m}$ wavelength range in order to determine the effective temperature of Arcturus.

Concerning the gravity, one notices that some publications give quite a different gravity w.r.t. our deduced value (with a difference $>0.6$ dex compared to $\log g=1.50$ deduced from the ISO-SWS and FTS-KP spectra). The large wavelength range of ISO-SWS, with the presence of many molecules, has proven to be very useful for the determination of the gravity for the cool giants. Our deduced value of $\log g=1.50$ is also corroborated by the excellent agreement between the FTSKP-spectrum and the synthetic spectrum of $\alpha$ Boo discussed in Paper II. Using, e.g., a gravity of $\log g=1.75$, leads to a gravity-inferred mass of $M_{\mathrm{g}}=1.30 M_{\odot}$. The evolutionary tracks of Fagotto et al. (1994) give also support to a mass value around $0.8 M_{\odot}\left(\right.$ for $T_{\text {eff }}=4300 \mathrm{~K}$ and $L=196 L_{\odot}$ ), suggesting the lower gravity value of $\log g=1.50$.

All obtained values for the metallicity mention $[\mathrm{Fe} / \mathrm{H}] \approx$ -0.50 . For the microturbulent velocity, a larger range occurs, with a minimum of $1.5 \mathrm{~km} \mathrm{~s}^{-1}$ (Fernández-Villaca nas et al. 1990; Aoki \& Tsuji 1997) and a maximum of $2.5 \mathrm{~km} \mathrm{~s}^{-1}$ (Gratton 1985). From studies on granulations, it is clear that the use of a single parameter to represent the non-thermal velocity field is highly phenomenological and thus, it is not surprising that large differences in deduced values for the microturbulence are obtained. 

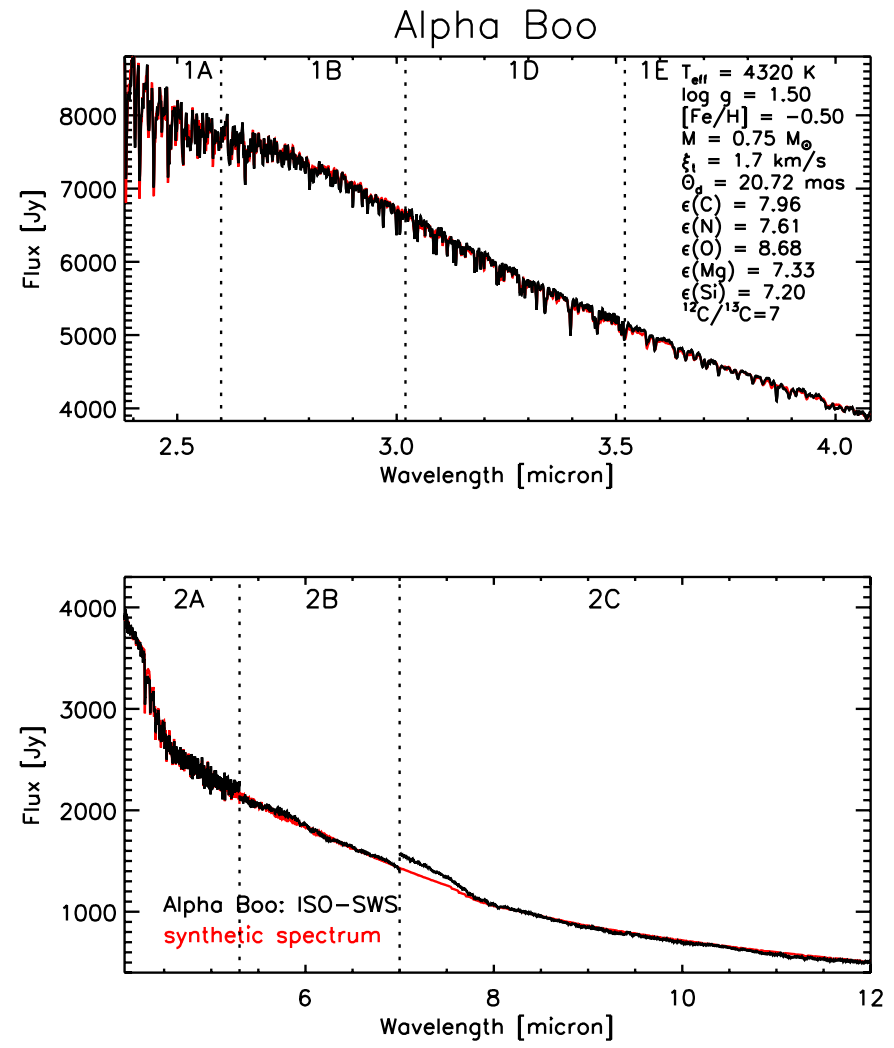

Fig. 5. Comparison between band 1 and band 2 of the ISO-SWS data (rev. 452) of $\alpha$ Boo (black) and the synthetic spectrum (grey) with stellar parameters $T_{\text {eff }}=4320 \mathrm{~K}, \log g=1.50, M=0.75 M_{\odot}$, $[\mathrm{Fe} / \mathrm{H}]=-0.50, \xi_{\mathrm{t}}=1.7 \mathrm{~km} \mathrm{~s}^{-1},{ }^{12} \mathrm{C} /{ }^{13} \mathrm{C}=7, \varepsilon(\mathrm{C})=7.96, \varepsilon(\mathrm{N})=$ $7.61, \varepsilon(\mathrm{O})=8.68, \varepsilon(\mathrm{Mg})=7.33, \varepsilon(\mathrm{Si})=7.20$ and $\theta_{\mathrm{d}}=20.72$ mas. $\mathrm{A}$ coloured version of this plot is available in the appendix as Fig. B.5.

For the carbon abundance, most of the studies give [C/Fe] being solar or somewhat sub-solar $(\leq 0.005)$, with the only exception being Altas (1987), who deduced an abnormal high carbon abundance using the Griffin atlas (Griffin 1968) and the model derived by Mäckle et al. (1975). Also for the nitrogen abundance, the value mentioned by Altas (1987) exceeds the other deduced values, although here the spread is higher than for the carbon abundance. We obtained $\varepsilon(\mathrm{O})$ using $\mathrm{OH}$-lines. Our deduced value agrees (within the error bar) with the ones obtained by Lambert \& Ries (1981), Kjærgaard et al. (1982), Altas (1987) and Peterson et al. (1993). Only the value obtained by Mäckle et al. (1975) is substantially lower than our value. Within the error bars, our ${ }^{12} \mathrm{C} /{ }^{13} \mathrm{C}$ ratio agrees with other published values.

Angular diameter, luminosity and radius values are in good agreement with other values found in the literature.

Quite a few authors have estimated the gravity-inferred mass, e.g. Mäckle et al. (1975), Gratton (1985), Harris et al. (1988), Judge \& Stencel (1991), Peterson et al. (1993). Differences in assumed/ adopted/ deduced gravity, radius, angular diameter or parallax value explain the observed differences in $M_{\mathrm{g}}$.

In general, the atmospheric parameters we derived for $\alpha$ Boo agree with the ones found by Peterson et al. (1993). They have obtained the stellar parameters by computing a model atmosphere which reproduces the observed flux distribution of the Griffin atlas (Griffin 1968) of $\alpha$ Boo. Accurate model atmospheres, opacities, line lists, ... are indispensable for a good assessment of the stellar parameters. Minor inaccuracies in photometric calibration formulae, in broadening parameters, in the assumptions made in the theoretical modelling computations, ... may lead to statistically relevant departures of the derived parameters. One should be careful when ascribing an atmospheric parameter based on the spectral type (like done by, e.g., Cohen et al. 1996). This is already obvious when comparing the parameters of $\alpha$ Boo and $\xi$ Dra, which are both K2 giants.

\section{6. $\alpha$ Tuc: AOT01, speed 4, revolution 866}

\subsubsection{Comparison between the ISO-SWS spectrum and the synthetic spectrum (Fig. 6)}

The somewhat higher $\beta$-value in band $1 \mathrm{~A}$ is partially due to a uncertainties in the temperature structure in the outer layers (Paper II) and partially due to the higher standard deviation in band 1A. The atomic features around $3.85 \mu \mathrm{m}$ and $3.98 \mu \mathrm{m}$ remain visible (see Fig. 3 in Paper II). The $\mathrm{OH}$ features are somewhat more pronounced than for $\alpha$ Boo. The $\mathrm{SiO}$ fundamental and first-overtone band, together with the CO fundamental band, arise in band 2 .

\subsubsection{Comparison with other published stellar parameters (see Table D. 4 in the appendix)}

Only a few published values for the different stellar parameters are available. The temperature derived from the ISOSWS spectrum and the $V-K$ colour is somewhat higher than the effective temperature derived from the $(V-R)$ colour index (Basri \& Linsky 1979; Pasquini \& Brocato 1992) and from other broad-band photometry (Glebocki \& Stawikowski 1988). Using the Johnson $B, V, R$ and $I$ colour measurements from Mendoza (1969) as listed by Simbad and the colourtemperature relations of McWilliam (1990), an effective temperature of $4080 \mathrm{~K}$ is obtained for $\alpha$ Tuc. The used $K$ magnitude of -0.09 mag listed by Wamsteker (1981) could not be checked against other literature values, since no other values were found.

There is however a large difference between the two listed gravities. Glebocki \& Stawikowski (1988) have estimated the mass from the mass-function and the position of the primary on the evolutionary track calculations. The radius $R$ was estimated from the bolometric magnitude and the effective temperature deduced from infrared broad-band photometry. Pasquini $\&$ Brocato (1992) have not taken the binary character of $\alpha$ Tuc into account, but have deduced the gravity from $M_{\mathrm{bol}}$ and a mass inferred from the comparison with evolutionary tracks. Due to a raw division of the stellar masses into four sub-groups, their gravity is the parameter containing the major uncertainties, which explains the difference with our first-guess value for $\log g$, derived from the same $M_{\mathrm{bol}}$ value, but using another $M_{\mathrm{hr}}$ value. From the ISO-SWS spectrum and the corresponding $\beta$ values, we could draw the conclusion that a gravity of 1.90 is too high. 

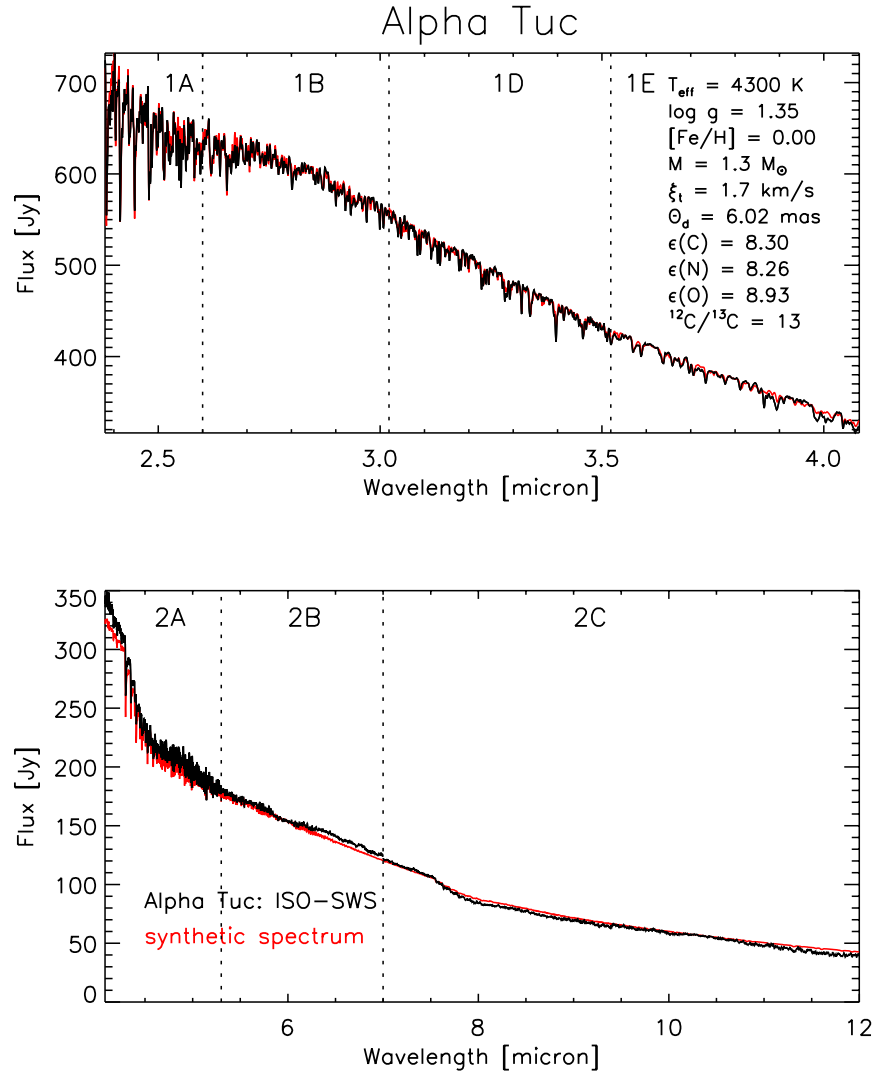

Fig. 6. Comparison between band 1 and band 2 of the ISO-SWS data (rev. 866) of $\alpha$ Tuc (black) and the synthetic spectrum (grey) with stellar parameters $T_{\text {eff }}=4300 \mathrm{~K}, \log g=1.35, M=1.3 M_{\odot},[\mathrm{Fe} / \mathrm{H}]=$ $0.00, \xi_{\mathrm{t}}=1.7 \mathrm{~km} \mathrm{~s}^{-1},{ }^{12} \mathrm{C} /{ }^{13} \mathrm{C}=13, \varepsilon(\mathrm{C})=8.30, \varepsilon(\mathrm{N})=8.26, \varepsilon(\mathrm{O})=$ 8.93 and $\theta_{\mathrm{d}}=6.02$ mas. A coloured version of this plot is available in the appendix as Fig. B.6.

Our deduced angular diameter of $6.02 \pm 0.37$ mas corresponds with the value given by Basri \& Linsky (1979), who have used a relation which links the colour $V-R$ to the angular diameter. These two values are higher than the value mentioned by Stencel et al. (1980). Both Basri \& Linsky (1979) and Stencel et al. (1980) have used the scaling laws described by Barnes \& Evans (1976) and Barnes et al. (1976) to convert fluxes observed on the Earth to fluxes at the stellar surface. Since this scaling law is based on the photometric colour $(V-R)$, an uncertainty in $(V-R)$ can induce a significant error. The photometric colour $(V-R)$ of $\alpha$ Tuc has been quoted as uncertain in both papers $((V-R)=0.9$ in Stencel et al. 1980 and $(V-R)=1.04$ in Basri \& Linsky 1979), explaining the large difference in $\theta_{\mathrm{d}}$.

The high-quality ISO-SWS spectra of $\alpha$ Tuc gave us the possibility to determine for the first time the $\mathrm{C}, \mathrm{N}$ and $\mathrm{O}$ abundances and the ${ }^{12} \mathrm{C} /{ }^{13} \mathrm{C}$-ratio for this target.

\section{7. $\beta$ UMi: AOT01, speed 4, revolution 182}

\subsubsection{Comparison between the ISO-SWS spectrum and the synthetic spectrum (Fig. 7)}

Band 1A has the largest $\beta$-value. For band 1A, the same reasons as for the previous stars can be quoted. The discrepancy between 3.91 and $3.97 \mu \mathrm{m}$ causes the somewhat larger $\beta_{1 \mathrm{E}^{-}}$ value. One can notify an analogous discrepancy in Fig. A.4 in the appendix. The pointing offset $\left(|\mathrm{d} y|>1^{\prime \prime}\right)$, together with a somewhat larger standard deviation after rebinning for the speed-4 observation in that wavelength range, render this discrepancy not significant. The $\mathrm{OH}$ spectral features dominate band $1 \mathrm{D}$ and band $1 \mathrm{E}$, while the first-overtone band of $\mathrm{SiO}$ is clearly visible around $4 \mu \mathrm{m}$. The $\mathrm{CO}$ fundamental and $\mathrm{SiO}$ fundamental and first-overtone bands remain visible in band 2 .

\subsubsection{Comparison with other published stellar parameters (see Table D.5 in the appendix)}

The published temperature values all agree very well, the only exception being the value derived by Lambert \& Ries (1981) and used by some other authors. As quoted by Ries (1981), Harris et al. (1988) and Luck \& Challener (1995), the effective temperature and gravity derived by Lambert \& Ries (1981) are too high and should be lowered by $\sim 240 \mathrm{~K}$ and $\sim 0.40$ dex respectively. Taking this remark into account and knowing that the gravity values quoted by Burnashev (1983) differ often with other values (see Sect. 3.3.2), our deduced gravity is in good agreement, although being at the lower end, with other deduced gravity values. The metallicity and microturbulent velocity agree with the other values listed in Table D.5. The ${ }^{12} \mathrm{C} /{ }^{13} \mathrm{C}$ ratio derived from the ISO-SWS spectrum is somewhat lower than other published values, but the difference is not significant due to the large standard deviation and the other problems discussed in Paper II for band 1A. The angular diameter from the SWS-spectrum lies in between the values quoted by Judge \& Stencel (1991) and Stencel et al. (1980). Being somewhat larger than the value given by Judge \& Stencel (1991), our deduced values of $R, M_{\mathrm{g}}$ and $L$ are also higher than those listed by Judge \& Stencel (1991).

\section{8. $\gamma$ Dra: AOT01, speed 4, revolution 377}

\subsubsection{Comparison between the ISO-SWS spectrum and the synthetic spectrum (Fig. 8)}

It was difficult to decide between $\log g \approx 1.20$ and $\log g \approx$ 1.55 for $\gamma$ Dra, since the Kolmogorov-Smirnov test - applied in the region from $2.8-3.2 \mu \mathrm{m}$, where the synthetic spectrum is quite sensitive to the gravity - yields almost the same $\beta$-value. Since a gravity of $\log g=1.30$ was obtained from $K, B C_{K}$, $\pi, T_{\text {eff }}(V-K)$ and $M_{\mathrm{hr}}$, this gravity was chosen to represent the "true" gravity of the star. The small difference between the two $\beta$-values is reflected in the higher uncertainty of $\log g$ in Table 3. The other comments for $\gamma$ Dra are similar to the ones for $\alpha$ Boo.

\subsubsection{Comparison with other published stellar parameters}

Different methods like IRFM, colour indices, interferometry, the Engelke function (Engelke 1992), ... almost all yield the same effective temperature for $\gamma$ Dra. The value given by 

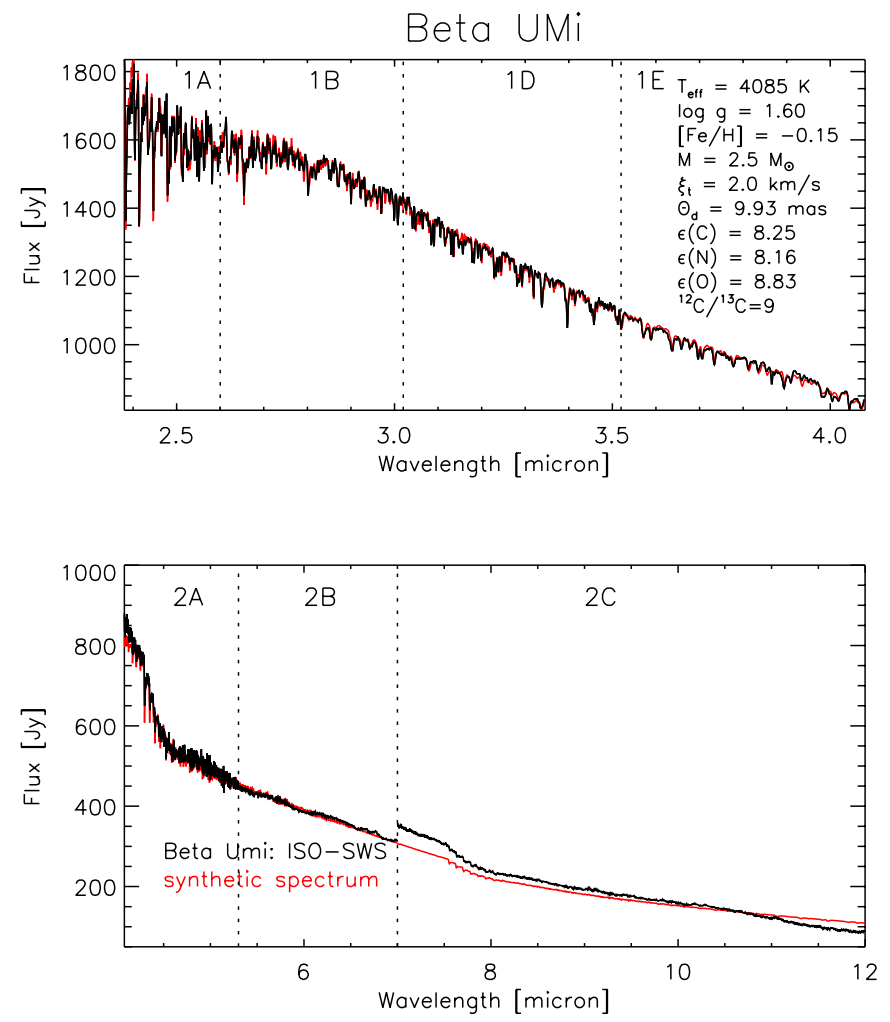

Fig. 7. Comparison between band 1 and band 2 of the ISO-SWS data (rev. 182) of $\beta$ UMi (black) and the synthetic spectrum (grey) with stellar parameters $T_{\text {eff }}=4085 \mathrm{~K}, \log g=1.60, M=2.50 M_{\odot}$, $[\mathrm{Fe} / \mathrm{H}]=-0.15, \xi_{\mathrm{t}}=2.0 \mathrm{~km} \mathrm{~s}^{-1},{ }^{12} \mathrm{C} /{ }^{13} \mathrm{C}=9, \varepsilon(\mathrm{C})=8.25, \varepsilon(\mathrm{N})=$ $8.16, \varepsilon(\mathrm{O})=8.83$ and $\theta_{\mathrm{d}}=9.93$ mas. A coloured version of this plot is available in the appendix as Fig. B.7.

Tomkin et al. (1975) is quite low, which may be due to inaccurate colour indices or calibration. We note that Faucherre et al. (1983), who derived effective temperatures from interferometric measurements of angular diameters, underestimated the effective temperature of $\delta$ Dra compared to our value, but for $\gamma$ Dra obtained a value slightly higher than ours. As for $\alpha$ Boo, the gravity deduced by Cohen et al. (1996) from the spectral type seems to be erroneous.

The reference quoted by Harris \& Lambert (1984) for the determination of the atmospheric parameters could not be traced back. The only two other deduced values for the gravity listed in Table D.6 are $\log g \simeq 1.55$ and $\log g \simeq 1.20$. As described in previous paragraph, the $\beta$-values from the Kolmogorov-Smirnov test for these two gravity values are quite alike.

Our deduced value for the angular diameter $\theta_{\mathrm{d}}=9.98 \pm$ $0.63 \mathrm{mas}$ is in close agreement with the values obtained by using the IRFM method (Blackwell \& Shallis 1977; Leggett et al. 1986; Bell \& Gustafsson 1989) or by using the Engelke function as was done by Cohen et al. (1996). While our results and the values adopted by Robinson et al. (1998) for the stellar radius are based on the Hipparcos' parallax and the deduced (or adopted) angular diameter, Leggett et al. (1986) used the parallax value given by Jenkins (1963) ( $\pi=17 \pm 6$ mas $)$. Consequently, their deduced radius (and luminosity) is higher than ours.
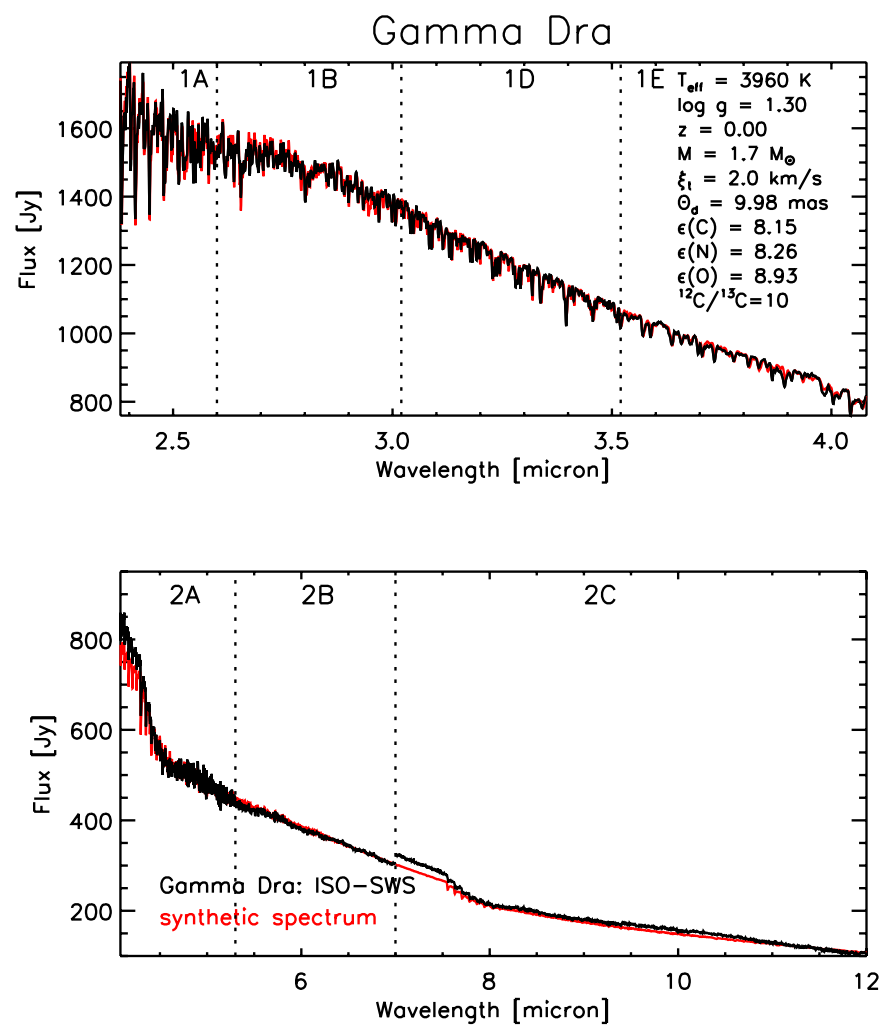

Fig. 8. Comparison between band 1 and band 2 of the ISO-SWS data (rev. 377) of $\gamma$ Dra (black) and the synthetic spectrum (grey) with stellar parameters $T_{\text {eff }}=3960 \mathrm{~K}, \log g=1.30, M=1.7 M_{\odot},[\mathrm{Fe} / \mathrm{H}]=$ $0.00, \xi_{\mathrm{t}}=2.0 \mathrm{~km} \mathrm{~s}^{-1},{ }^{12} \mathrm{C} /{ }^{13} \mathrm{C}=10, \varepsilon(\mathrm{C})=8.15, \varepsilon(\mathrm{N})=8.26, \varepsilon(\mathrm{O})=$ 8.93 and $\theta_{\mathrm{d}}=9.98$ mas. A coloured version of this plot is available in the appendix as Fig. B.8.

Only Harris et al. (1988) and Robinson et al. (1998) have estimated a gravity-inferred mass from adopted $g$ and $R$ values. The value of Robinson et al. (1998) is higher than our derived $M_{\mathrm{g}}$ value due to the use of a higher gravity, while the value obtained by Harris et al. (1988) $\left(M=0.9 M_{\odot}\right)$ is much lower. This low value was discarded by Harris et al. (1988), who instead adopted $M=2.0 M_{\odot}$.

$\mathrm{C}, \mathrm{N}$ and $\mathrm{O}$ abundances agree fairly well with the ones obtained by Lambert \& Ries (1981).

\section{9. $\alpha$ Tau: AOT01, speed 4, revolution 636}

$\alpha$ Tau has been discussed in Paper I.

\subsection{H Sco: AOT01, speed 4, revolution 847}

\subsubsection{Comparison between the ISO-SWS spectrum and the synthetic spectrum (Fig. 9)}

The use of the initial values for the effective temperature and gravity of $T_{\text {eff }}=3750 \mathrm{~K}$ and $\log g=1.00$ resulted in synthetic spectrum being very discrepant from the ISO-SWS spectrum. From the slope of the ISO-SWS data between 2.8 and $4.08 \mu \mathrm{m}$, the effective temperature was found to be higher by $\sim 150 \mathrm{~K}$. The logarithm of the gravity had to be increased with 0.3 dex. The used $K$ magnitude to derive the initial values of $T_{\text {eff }}$ and 
$\log g$ was quoted to be uncertain (Price 1968), which may result in initial values being quite off.

From $\mathrm{H}$ Sco on, the spectra are dominated by water lines. It has been calculated that at a temperature of about $3900 \mathrm{~K}$ the concentrations of water and $\mathrm{OH}$ are equal; below this temperature, water is the dominant oxygen-containing molecule as well as the dominant opacity source (Wallace et al. 1995).

\subsubsection{Comparison with other published stellar parameters}

HD 149447 is classified by Simbad as a K6 III giant. Perrin et al. (1998) estimated the effective temperature for a K5III giant (with $V-K=3.52$ ) as being $T_{\text {eff }}=3980 \mathrm{~K}$ and for an M0 III giant (with $V-K=3.78$ ) as $T_{\text {eff }}=3895 \mathrm{~K}$. Gray (1992) has, however, listed somewhat lower temperatures, i.e. $T_{\text {eff }}=3915 \mathrm{~K}$ for a K5 III giant and $T_{\text {eff }}=3726 \mathrm{~K}$ for an M0 III giant. Using the Johnson $B, V, R$ and $I$ colour measurements listed by Johnson et al. (1966) and the colour-temperature relations of McWilliam (1990), we derived an effective temperature $T_{\text {eff }} \approx 3900 \mathrm{~K}$. These $T_{\text {eff }}$ values support our $T_{\text {eff }}$ value estimated from the ISO-SWS spectrum of H Sco.

The quantities $\log g$, mass, $\xi_{\mathrm{t}},[\mathrm{Fe} / \mathrm{H}], \mathrm{CNO}$-abundances, ${ }^{12} \mathrm{C} /{ }^{13} \mathrm{C}$-ratio, $\theta_{\mathrm{d}}, L$ and $R$ are here derived for the first time for this stellar source.

\subsection{1. $\beta$ And: AOT01, speed 4, revolution 795}

\subsubsection{Comparison between the ISO-SWS spectrum and the synthetic spectrum (Fig. 10)}

Just like the K-giants, this "normal" M-giant displays the molecular features and the discrepancies summarised in Paper I. However, $\mathrm{H}_{2} \mathrm{O}$, rather than $\mathrm{OH}$, has now become the main absorber in the wavelength region from $2.8 \mu \mathrm{m}$ to $4 \mu \mathrm{m}$, although the $\mathrm{OH}$ lines remain very well visible.

\subsubsection{Comparison with other published stellar parameters (see Table 10 in the appendix)}

As for $\gamma$ Dra and $\alpha$ Tau, the effective temperatures given by Tomkin et al. (1975); Linsky \& Ayres (1978); Scargle \& Strecker (1979) are quite low compared to the other values derived from different methods. The interferometric value by Dyck et al. (1998) is the highest $T_{\text {eff }}$ value in Table D.7.

From $K, B C_{K}, \pi, T_{\mathrm{eff}}(V-K)$ and $M_{\mathrm{hr}}$ an initial input gravity of $\log g=0.95$ was derived. Using this gravity value, $T_{\text {eff }}=3880 \mathrm{~K}, \xi_{\mathrm{t}}=2.0 \mathrm{~km} \mathrm{~s}^{-1},[\mathrm{Fe} / \mathrm{H}]=0.00, \varepsilon(\mathrm{C})=8.12$, $\varepsilon(\mathrm{N})=8.37, \varepsilon(\mathrm{O})=9.08$ and ${ }^{12} \mathrm{C} /{ }^{13} \mathrm{C}=9$, a good agreement with the observed ISO-SWS spectrum was obtained. This gravity value of $\log g=0.95$ is, however, much lower than most of the other quoted values in Table D.7. Examining the other gravity values, we see that

- Clegg et al. (1979) have derived log $g$ from an assumed mass of $1 M_{\odot}$, a $T_{\text {eff }}$ value derived from spectral type and colours and have estimated a luminosity value using the HR diagram of Scalo (1976). Our deduced $M_{\mathrm{hr}}$ of
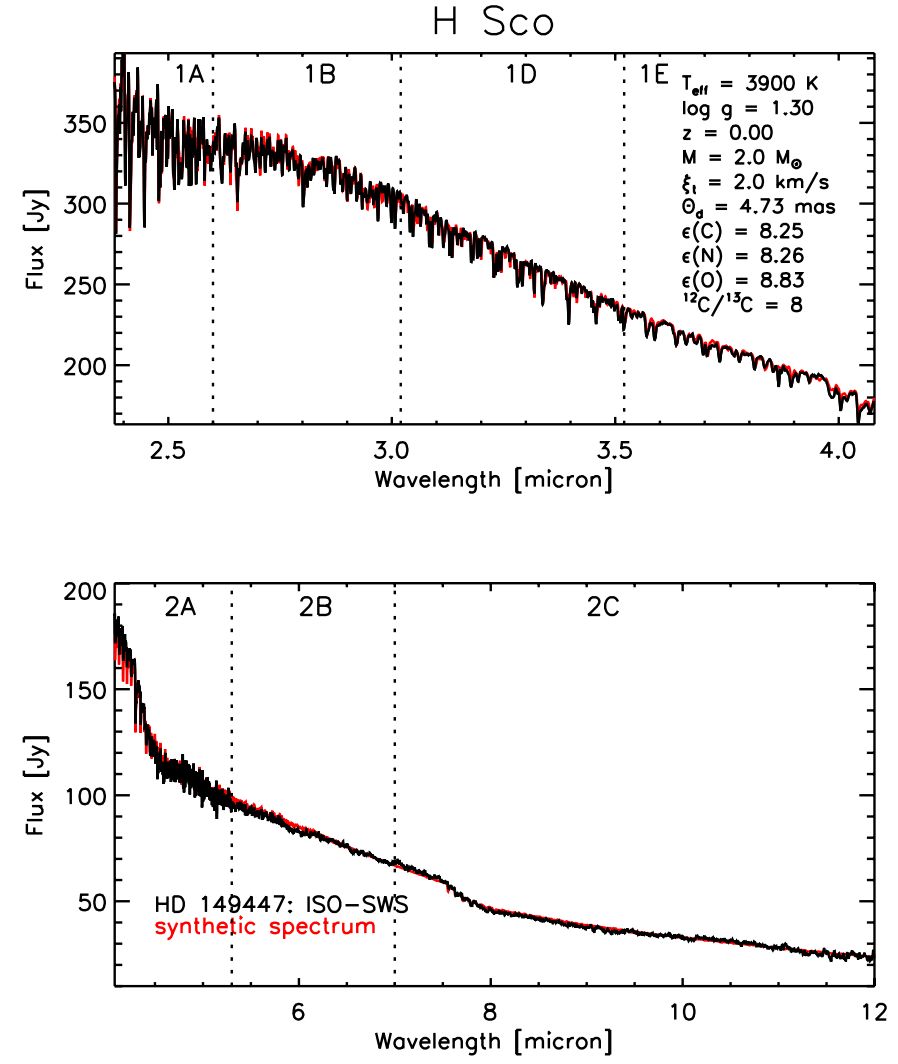

Fig. 9. Comparison between band 1 and band 2 of the ISO-SWS data (rev. 847) of H Sco (black) and the synthetic spectrum (grey) with stellar parameters $T_{\text {eff }}=3900 \mathrm{~K}, \log g=1.30, M=2.0 M_{\odot},[\mathrm{Fe} / \mathrm{H}]=$ $0.00, \xi_{\mathrm{t}}=2.0 \mathrm{~km} \mathrm{~s}^{-1},{ }^{12} \mathrm{C} /{ }^{13} \mathrm{C}=8, \varepsilon(\mathrm{C})=8.20, \varepsilon(\mathrm{N})=8.26, \varepsilon(\mathrm{O})=$ 8.83 and $\theta_{\mathrm{d}}=4.73$ mas. A coloured version of this plot is available in the appendix as Fig. B.9.

$2.2 M_{\odot}$ would result in a higher luminosity and thus in a lower gravity.

- Both Smith \& Lambert (1985) and Lazaro et al. (1991) have estimated the effective temperature from the $V-K$ colour and the luminosity from $M_{V}(K)$ obtained via the WilsonBappu effect and $B C_{K}$. Comparing, however, these $M_{V}(K)$ values from Wilson (1976) with $M_{V}$ values obtained using the $V$ colour given by Simbad and the Hipparcos' parallax, we note that these $M_{V}(K)$ are systematically higher than our derived $M_{V}$ values (with a mean difference of $0.73 \mathrm{mag}$ ). Using another sample of cool giants, Tsuji (1981) compared the results from the Wilson-Bappu effect with those by Eggen (1973), showing that the results from the WilsonBappu effect are systematically $\sim 0.5 \mathrm{mag}$ fainter. Hence, Tsuji (1981) decided to use the mean of the two values. Using too high an $M_{\text {bol }}$ value (by $\sim 0.73 \mathrm{mag}$ ) results in too high a $\log g$ value (by $\sim 0.3 \mathrm{dex}$ ). Moreover, taking these questionable values for the luminosity into account, the mass values estimated by Smith \& Lambert (1985) and Lazaro et al. (1991) using evolutionary tracks are meaningless. In addition, Lazaro et al. (1991) have estimated stellar parameters per spectral type, and so have adopted a mean mass of $1.5 M_{\odot}$. Although having the same spectral type, stellar objects may differ significantly in stellar parameters. 

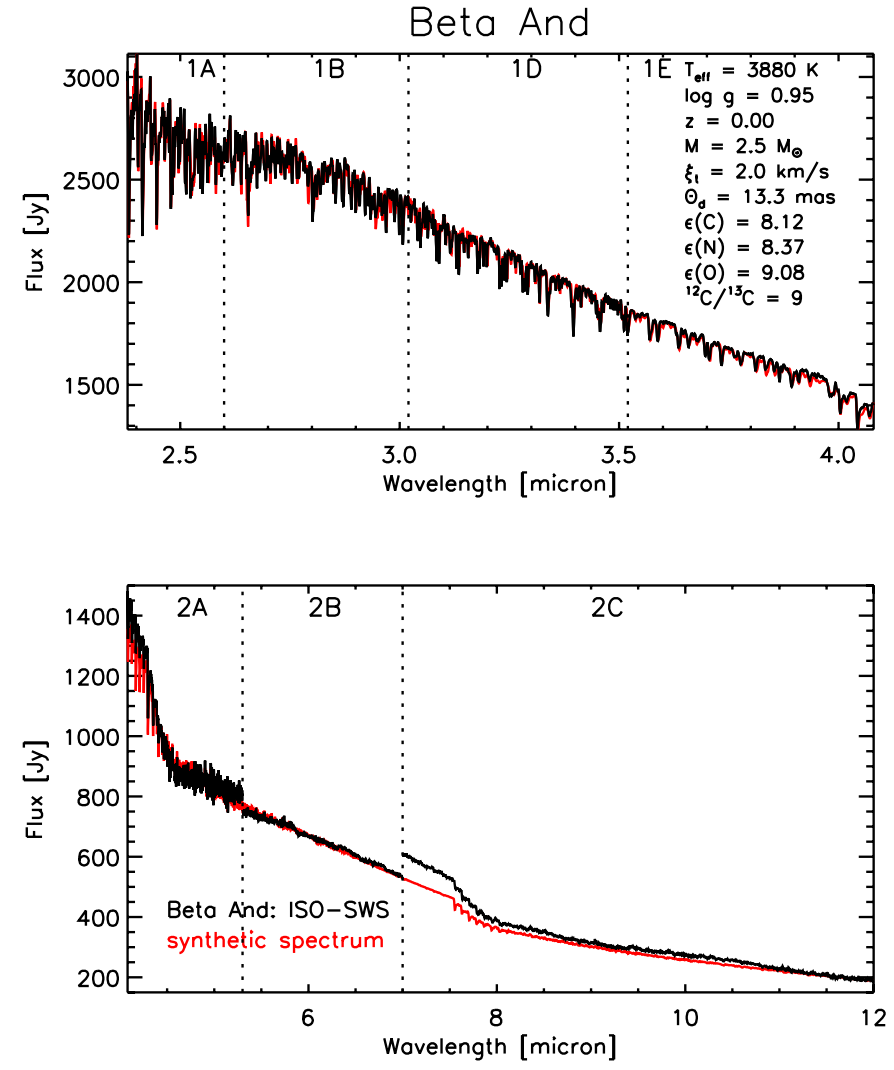

Fig. 10. Comparison between band 1 and band 2 of the ISO-SWS data (rev. 795) of $\beta$ And (black) and the synthetic spectrum (grey) with stellar parameters $T_{\text {eff }}=3880 \mathrm{~K}, \log g=0.95, M=2.5 M_{\odot},[\mathrm{Fe} / \mathrm{H}]=$ $0.00, \xi_{\mathrm{t}}=2.0 \mathrm{~km} \mathrm{~s}^{-1},{ }^{12} \mathrm{C} /{ }^{13} \mathrm{C}=9, \varepsilon(\mathrm{C})=8.12, \varepsilon(\mathrm{N})=8.37, \varepsilon(\mathrm{O})=$ 9.08 and $\theta_{\mathrm{d}}=13.30$ mas. A coloured version of this plot is available in the appendix as Fig. B.10.

- Judge \& Stencel (1991) have estimated the gravity from photospheric line studies, but these results could not be traced back.

Thus, we may conclude that the other quoted $\log g$ values in Table D.7 are either too high or are meaningless.

The derived angular diameter tallies with other indirectly determined angular diameters given in Table D.7. The use of old parallax values by Faucherre et al. (1983), Volk \& Cohen (1989), Judge \& Stencel (1991) - which may be almost a factor 3 higher than the Hipparcos' parallax - resulted, however, in a radius value much higher than any literature values. As a consequence, our deduced luminosity exceeds the other quoted values. Since our deduced gravity is at the lower end of the other quoted gravity values, our deduced C-abundance is also somewhat lower (see Paper I). The abundances of nitrogen and oxygen agree with other quoted values.

\subsection{2. $\alpha$ Cet: AOT01, speed 4, revolution 797}

\subsubsection{Comparison between the ISO-SWS spectrum and the synthetic spectrum (Fig. 11)}

For this cool giant, the spectrum resulting from the computations with a standard stellar model, i.e. without a stellar wind, shows a very good agreement with the ISO-SWS spectrum of $\alpha$ Cet. The discrepancies described in Paper I occur also for $\alpha$ Cet. The small atomic feature around $3.85 \mu \mathrm{m}$ remains marginally visible.

\subsubsection{Comparison with other published stellar parameters (see Table D. 8 in the appendix)}

Napiwotzki et al. (1993) quoted that the resulting IRFM temperatures are too low by $1.6-2.8 \%$, but the effective temperature found by Tsuji (1981) is $\sim 160 \mathrm{~K}$ higher than the effective temperature deduced from the $V-K$ colour and confirmed by the ISO-SWS data. Using improved $\mathrm{H}^{-}$opacity, Blackwell et al. (1991) derived an effective temperature from the IRFM being $3745 \mathrm{~K}$, which matches our derived temperature value.

Concerning the quoted gravity values in Table D.8, the same comments can be given as for $\beta$ And. The use of too low a luminosity value results in too high a gravity value (Clegg et al. 1979; Lazaro et al. 1991; Tsuji 1986). Moreover, assuming a stellar mass $\left(1 M_{\odot}\right.$ by Clegg et al. (1979), $3 M_{\odot}$ by Tsuji (1986), $1.5 M_{\odot}$ by Lazaro et al. (1991) based on the spectral type, can introduce significant errors on the gravity. The obtained value by Judge \& Stencel (1991) could not be traced back. Cohen et al. (1996) used a statistical relation between spectral type and gravity which may result in inaccurate gravity values taking into account the divergent stellar parameters for different targets having the same spectral type (cf. e.g. $\alpha$ Cet and $\beta$ Peg). Burnashev (1983) has derived gravity values from narrow-band photometric colours. As can be seen from Table D.8, the obtained values differ largely (see also the remarks given in Sects. 3.3.2 and 3.7.2). Hence, once more, we may conclude that the other quoted gravity values in Table D.8 are not accurate.

Our angular diameter deduced from the ISO-SWS spectrum agrees quite well with other angular diameters deduced using indirect methods (e.g. Blackwell et al. 1991; Judge \& Stencel 1991). Judge \& Stencel (1991) used a somewhat lower parallax value quoted by Jenkins (1952) (which differs by a factor $\sim 1.2$ with the Hipparcos' parallax), resulting in a higher radius and luminosity value than ours.

Our deduced carbon abundance differs somewhat from the values deduced by Tsuji (1991), who used secondovertone CO-lines. These values are higher than the values inferred from first-overtone CO-lines by Tsuji (1986) and Lazaro et al. (1991). Tsuji (1991) discussed this problem in conjunction with the smaller $\xi_{\mathrm{t}}$, deduced from the second-overtone, than from the first-overtone CO lines. Probably, the origin of the discrepancy between the carbon abundances derived from the more or less saturated lines of the $\mathrm{CO}$ first-overtone and those from the less saturated second-overtone lines can, at least partly, be explained by an over-simplification of the treatment of the microturbulent velocity, for which in classical models a depth-independent, isotropic Gaussian model is taken. Even in classical abundance analysis, an error of only $0.5 \mathrm{~km} \mathrm{~s}^{-1}$ in $\xi_{\mathrm{t}}$ produces an error as large as $0.3 \mathrm{dex}$ in the derived abundances. Since line asymmetries in cool giant stars are as large as $0.5 \mathrm{~km} \mathrm{~s}^{-1}$, a poor modelling of the turbulence may also 

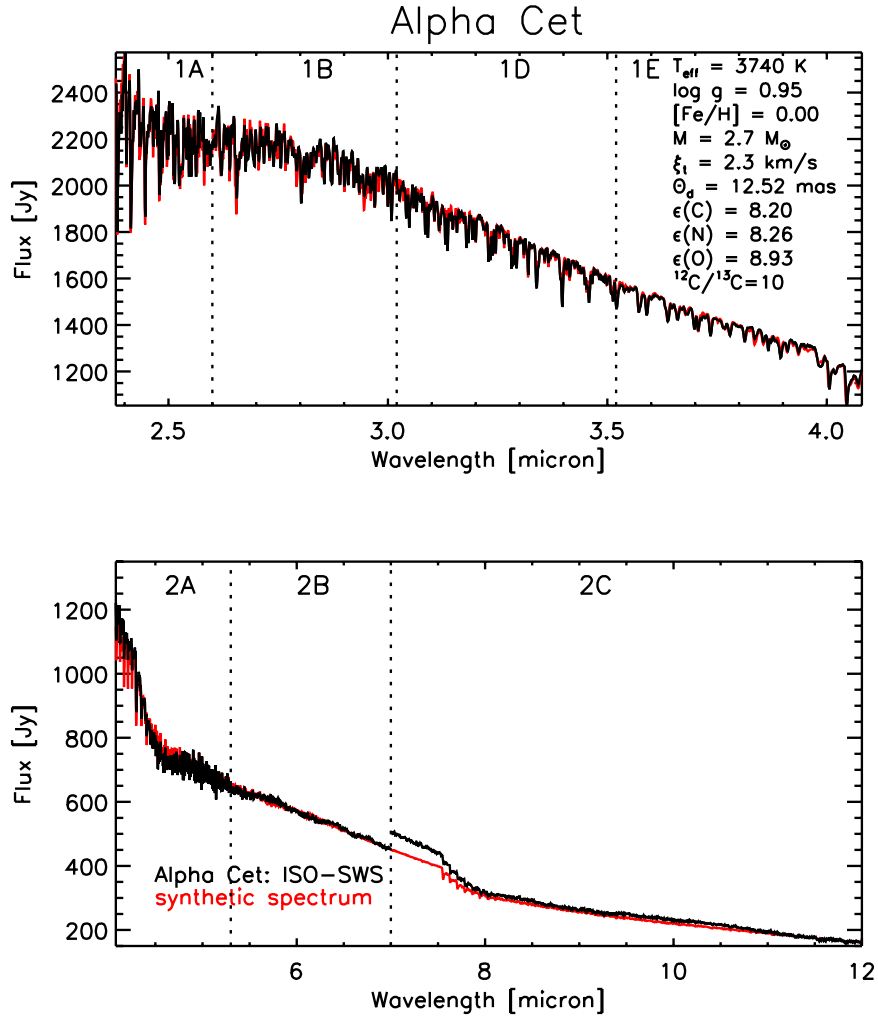

Fig. 11. Comparison between band 1 and band 2 of the ISO-SWS data (rev. 797) of $\alpha$ Cet (black) and the synthetic spectrum (grey) with stellar parameters $T_{\text {eff }}=3740 \mathrm{~K}, \log g=0.95, M=2.7 M_{\odot},[\mathrm{Fe} / \mathrm{H}]=$ $0.00, \xi_{\mathrm{t}}=2.3 \mathrm{~km} \mathrm{~s}^{-1},{ }^{12} \mathrm{C} /{ }^{13} \mathrm{C}=10, \varepsilon(\mathrm{C})=8.20, \varepsilon(\mathrm{N})=8.26, \varepsilon(\mathrm{O})=$ 8.93 and $\theta_{\mathrm{d}}=12.52$ mas. A coloured version of this plot is available in the appendix as Fig. B.11.

produce similar or even larger errors on the abundances derived from saturated lines. Our carbon abundance is, however, deduced from first-overtone lines. Its higher value w.r.t. the value given by Tsuji (1986) may be explained by our lower $\xi_{\mathrm{t}}$. Moreover, when using the parameter values as deduced by Tsuji (1981, Tsuji 1986, Tsuji 1991 a bad correspondence is seen between the observed and synthetic spectra: when using $\varepsilon(\mathrm{C})=8.06$ the $\mathrm{CO}(\Delta v=2)$ lines are predicted as being far too weak, while they are predicted as being far too strong for $\varepsilon(C)=8.50$. It should also be noted that the agreement in band $1 \mathrm{~B}$ was very bad.

\subsection{3. $\beta$ Peg: AOT01, speed 4, revolution 551}

\subsubsection{Comparison between the ISO-SWS spectrum and the synthetic spectrum (Fig. 12)}

By using the classical models and synthetic spectra as calculated by the MARCS and TURBOSPECTRUM codes, it turned out impossible to determine stellar parameters from the ISO-SWS data of $\beta$ Peg: in the range $2.6-3.2 \mu \mathrm{m}$, certain lines were always predicted as far too weak (see, e.g., Fig. 12, where the synthetic spectra for 2 different sets of parameters are plotted).

Already from the early eighties, it was clear that the nearinfrared absorption lines of $\mathrm{CO}$ and $\mathrm{H}_{2} \mathrm{O}$ molecules yield insight into the structure of the inner parts of an expanding envelope (Tielens 1983). The picture emanating from these data was that three different regions are to be recognised:

(a) an extending pulsating photosphere terminated by

(b) a stationary layer which starts

(c) the cool expanding envelope.

In panel C in Fig. 12 - where the SWS observational data of $\beta$ Peg are divided by the synthetic spectrum specified in panel $\mathrm{B}$ - a clear pattern is appearing. Comparing this pattern with typical molecular absorption patterns as displayed e.g. in Fig. 3.5 in Decin (2000) or in Fig. 4 in Decin et al. (2000), we see that - at least $-\mathrm{H}_{2} \mathrm{O}$ is necessary to explain this excess absorption.

The discovery of such excess absorption/emission features visible in the ISO-SWS data was for the first time discussed by Tsuji et al. (1997). They concluded that these features could be interpreted as due to an excess absorption originating in a "warm molecular envelope". These layers are clearly distinct from the much cooler circumstellar envelopes (CSE) already known from radio observations. At that time, it was however still not clear how such a molecule forming region could be developed.

A similar structure of features was found from ISO observations in quite some other stars (Fig. 13), among them $\alpha$ Ori (Cami et al. 1997; Justtanont et al. 1998; de Jong 1999; Yamamura et al. 1999; Ryde et al. 1999). Together with $\mu$ Cep, $\alpha$ Ori is one of the two objects whose CSE kinetic temperature distribution has been analysed (Rodgers \& Glassgold 1991). Indications were found by other methods and different authors that a hydrostatic model is not able to model these stars correctly (e.g. Hinkle et al. 1982; Aringer et al. 1999). Such discrepancies, found by both models developed by Tsuji and collaborators and models developed by the Uppsala group, may be too large to be relaxed by improving the model of the photosphere. Dynamical models for the outer layers of oxygen-rich AGB-stars reveal, on the other hand, a layered structure of the molecules and much higher column densities than predicted by hydrostatic models (Woitke et al. 1999; Höfner et al. 2002). This is a natural consequence of the shock waves occurring in these models. The overall amount of levitated gas found in the models exceeds the lower density limits of $\mathrm{CO}, \mathrm{H}_{2} \mathrm{O}, \mathrm{CO}_{2}$ and $\mathrm{SO}_{2}$ inferred from the ISO-SWS observations. Therefore, the levitation of the outer atmospheres of AGB-stars by pulsations seems to be a possible mechanism to form these "warm molecular layers". Other effects, such as dust formation and chemical non-equilibrium, are probably also involved.

Thus, it is an established fact that a better understanding of the complicated structure of cool stars - and more precisely their spectral features around $3 \mu \mathrm{m}$ - will only be reached once dynamical modelling of stars, including good opacity calculations, NLTE and a CSE, are fully developed. One should therefore be very careful in the interpretation of results based on current modelling.

In order to determine $\varepsilon(\mathrm{C})$, the ${ }^{12} \mathrm{C} /{ }^{13} \mathrm{C}$-ratio, $\theta_{\mathrm{d}}, L, R$ and $M_{\mathrm{g}}$ we have adopted as $T_{\text {eff }}$ and $\log g$ the values obtained in Sect. 3.1. Furthermore, we adopted $[\mathrm{Fe} / \mathrm{H}]=$ $0.00, \xi_{\mathrm{t}}=2.0 \mathrm{~km} \mathrm{~s}^{-1}, \varepsilon(\mathrm{N})=8.18$ and $\varepsilon(\mathrm{O})=8.93$ assuming that $\beta$ Peg has already gone through the first dredge-up. For the computation of the upper and lower limit of the stellar mass, $\Delta \log g=0.40$ dex was assumed. 

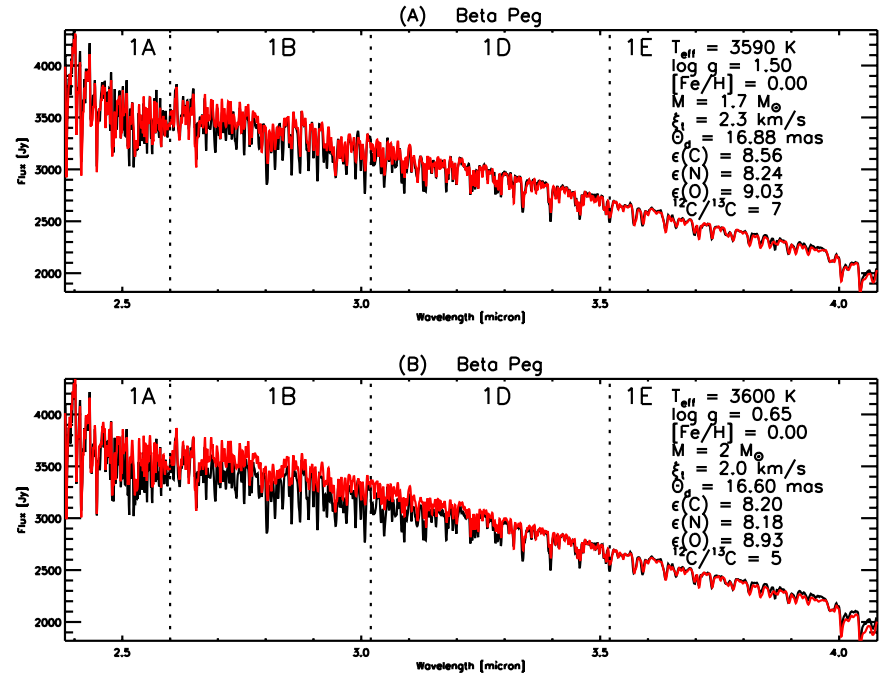

(C) SwS / synthetic of (B)

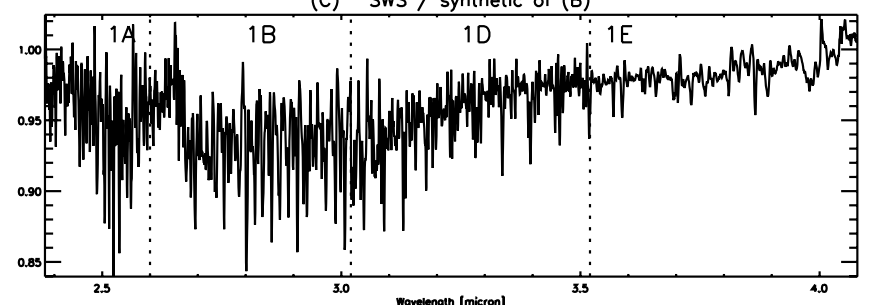

Fig. 12. Panel A): Comparison between band 1 of the ISO-SWS data (rev. 551) of $\beta$ Peg (black) and the synthetic spectrum (grey) with stellar parameters $T_{\text {eff }}=3590 \mathrm{~K}, \log g=1.50, M=1.7 M_{\odot},[\mathrm{Fe} / \mathrm{H}]=$ $0.00, \xi_{\mathrm{t}}=2.3 \mathrm{~km} \mathrm{~s}^{-1},{ }^{12} \mathrm{C} /{ }^{13} \mathrm{C}=7, \varepsilon(\mathrm{C})=8.56, \varepsilon(\mathrm{N})=8.24, \varepsilon(\mathrm{O})=$ 9.03 and $\theta_{\mathrm{d}}=16.88$ mas. Panel B): Comparison between band 1 of the ISO-SWS data (rev. 551) of $\beta$ Peg (black) and the synthetic spectrum (grey) with stellar parameters $T_{\text {eff }}=3600 \mathrm{~K}, \log g=0.65, M=2 M_{\odot}$, $[\mathrm{Fe} / \mathrm{H}]=0.00, \xi_{\mathrm{t}}=2.0 \mathrm{~km} \mathrm{~s}^{-1},{ }^{12} \mathrm{C} /{ }^{13} \mathrm{C}=5, \varepsilon(\mathrm{C})=8.20, \varepsilon(\mathrm{N})=8.18$, $\varepsilon(\mathrm{O})=8.93$ and $\theta_{\mathrm{d}}=16.60$ mas. Panel C): ISO-SWS observational data (rev. 551) of $\beta$ Peg divided by the synthetic spectrum with the same parameters as in panel B). A coloured version of this plot is available in the appendix as Fig. B.12.

\subsubsection{Comparison with other published stellar parameters(see Table D.9 in the appendix)}

As for $\alpha$ Boo and $\beta$ And, the highest effective temperature value for $\beta$ Peg in Table D.9 is given by the interferometric value of Dyck et al. (1998). The other $T_{\text {eff }}$ values do agree quite well.

A discussion on the other quoted gravity values in Table D. 9 can be found in the corresponding sections for $\beta$ And and $\alpha$ Cet. As for $\alpha$ Cet, Tsuji (1986) has determined $M_{\text {bol }}$ as being the mean of the value given by the Wilson-Bappu relation (Wilson 1976) $\left(M_{\mathrm{bol}}=-1.9\right)$ and the result found by Eggen (1973) $\left(M_{\mathrm{bol}}=-3.2\right)$. Since this mean value is higher than our value for $M_{\text {bol }}$ by $0.79 \mathrm{mag}$ (see Table $2, M_{\text {bol }}=-3.34$ ), the obtained value for $\log g$ is higher by -0.3 dex. Tsuji (1991) quoted, however, a lower gravity value, being $\log g=0.5 \mathrm{dex}$, but the reason for this decrease is not mentioned.

The microturbulence was determined by various authors. They have used different kinds of spectral lines, such as the $\mathrm{SiO}$ first-overtone lines (Lambert et al. 1987), the CO first-overtone lines (Tsuji 1986), the CO second-overtone lines (Tsuji 1991),

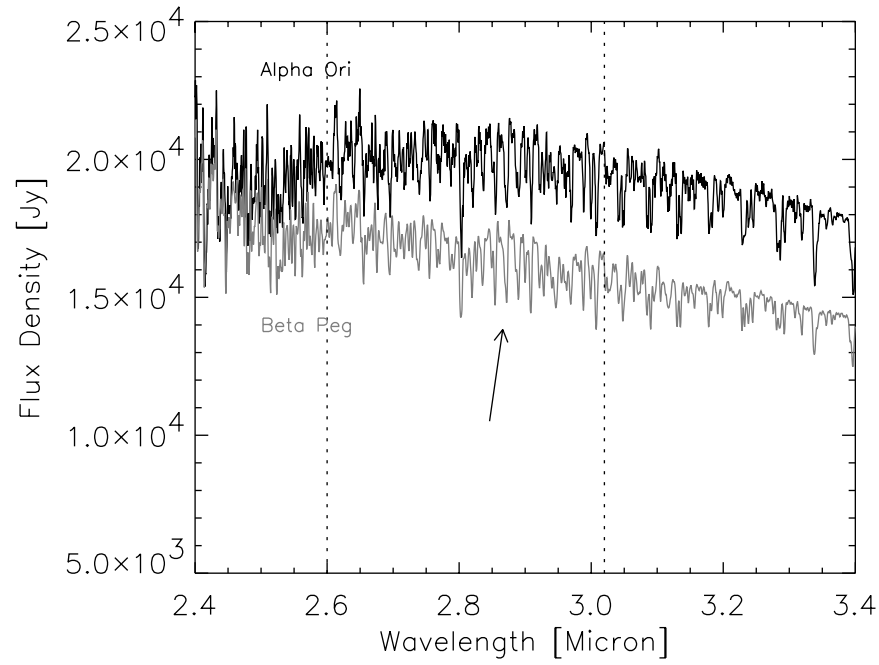

Fig. 13. $\alpha$ Ori and $\beta$ Peg are compared in the wavelength region from 2.4 to $3.4 \mu \mathrm{m}$. The flux values of $\beta$ Peg are multiplied by a factor 5. The "problematic" $\mathrm{OH} / \mathrm{H}_{2} \mathrm{O}$ lines are indicated by the arrow.

CN lines (Aoki \& Tsuji 1997), Fe I, Ni I and Ti I lines (Smith \& Lambert 1985). Unfortunately, these studies all yield different values.

Also for $\beta$ Peg, a large difference is visible between the carbon abundance deduced from the $\mathrm{CO}(\Delta v=2)$ lines and from the CO $(\Delta v=3)$ lines as obtained by Tsuji $(1986$, Tsuji 1991). Smith \& Lambert (1990) have used both CO $(\Delta v=2)$ and $\mathrm{CO}(\Delta v=3)$ lines. They are the only ones having deduced $\varepsilon(\mathrm{C})$, $\varepsilon(\mathrm{N})$ and $\varepsilon(\mathrm{O})$ simultaneously based on high-resolution spectra of $\mathrm{OH}(\Delta v=2), \mathrm{CO}(\Delta v=3), \mathrm{CO}(\Delta v=2)$ and $\mathrm{CN}(\Delta v=2)$ lines. Using the assumed stellar parameters as described in previous section, $\varepsilon(\mathrm{C}), \theta_{\mathrm{d}}, L, R, M_{\mathrm{g}}$ and the ${ }^{12} \mathrm{C} /{ }^{13} \mathrm{C}$-ratio are deduced from the ISO-SWS spectrum of $\beta$ Peg. We assigned a quite high error bar on the derived $\varepsilon(\mathrm{C})$ and ${ }^{12} \mathrm{C} /{ }^{13} \mathrm{C}$-ratio, since these ratios were deduced only from the first ${ }^{12} \mathrm{CO}$ and ${ }^{13} \mathrm{CO}$ features before $2.45 \mu \mathrm{m}$, where the spectrum is less disturbed by the excess absorption of $\mathrm{H}_{2} \mathrm{O}$. As for $\alpha$ Cet, the ${ }^{12} \mathrm{C} /{ }^{13} \mathrm{C}$-ratio obtained by Lazaro et al. (1991) is higher than the value deduced in this and other studies.

Our obtained angular diameter agrees well with the angular diameter obtained most recent by Blackwell et al. (1991) using IRFM. Also the luminosity and the radius correspond with other quoted values.

\section{Discussion on the derived stellar parameters}

\subsection{Temperatures derived from $V-K$ colours}

It is instructive to compare the ISO-SWS $T_{\text {eff }}$, mostly sensitive to the slope of the spectrum between 2.8 and $4 \mu \mathrm{m}$, to $T_{\text {eff }}$ derived by other means. As already said in Sect. 3.1, one can calculate $T_{\text {eff }}$ directly from $V-K$ using (semi-) empirical ( $T_{\text {eff }}$, $\left.(V-K)_{0}\right)$ temperature scales. Such calibrations are e.g. given by Ridgway et al. (1980), di Benedetto $(1993,1998)$ and Bessell et al. (1998) (see Sect. 3.1). Ridgway et al. (1980) have established an empirical effective temperature calibration by using angular diameters from lunar occultation in combination with 
infrared photometry. More recently, Michelson interferometry has been producing much more precise radii for the $\mathrm{K}$ and $\mathrm{M}$ stars (di Benedetto 1993, 1998).

$T_{\text {eff }}$ may also be derived from $m_{\text {bol }}$ and the angular diameter deduced from the ISO-spectra. It is easily shown that: $\log \left(T_{\text {eff }}\right)=2.720-0.5 \log \left(\theta_{\mathrm{D}} / 1000\right)-m_{\text {bol }} / 10$, with $\theta_{\mathrm{d}}$ in mas and assuming that $M_{\mathrm{bol}, \odot}=4.74$ (Bessell et al. 1998). From $m_{\text {bol }}\left(K_{0}, B C_{K}\left((V-K)_{0}\right)\right)$ in Table 2 and $\theta_{\mathrm{d}}$ from Table 3, $T_{\text {eff }}\left(K_{0}, B C_{K}, \theta_{\mathrm{D}}\right)$ is calculated. This $T_{\text {eff }}\left(K_{0}, B C_{K}, \theta_{\mathrm{D}}\right)$ is listed in Col. 6 in Table 5, together with $T_{\text {eff }}$ derived from the three $T_{\text {eff }}(V-K)$ calibrations discussed above. The comparison to $T_{\text {eff }}$ (IS0) (Fig. 14) shows that these methods do agree within the $\sim 5 \%$ level, with the exception being $\mathrm{H}$ Sco (see further discussion)! Only for a few stars, $T_{\text {eff }}$ (ISO) has been adjusted slightly w.r.t. the first guess value $T_{\text {eff }}\left((V-K)_{0}\right)$ derived from Bessell et al. (1998). This good general agreement in $T_{\text {eff }}$ proves that $T_{\text {eff }}$ as derived from ISO-SWS spectra is compatible with $T_{\text {eff }}$ derived from photometry. Since all the $T_{\text {eff }}\left((V-K)_{0}\right)$ calibrations are based on almost the same principle, it is normal that all the $T_{\text {eff }}\left((V-K)_{0}\right)$ values are clustered together w.r.t. $T_{\text {eff }}\left(K_{0}, B C_{K}, \theta_{\mathrm{D}}\right)$, as is seen in the lowermost plot of Fig. 14. For H Sco and $\alpha$ Tuc, the derived $T_{\text {eff }}\left(K_{0}, B C_{K}, \theta_{\mathrm{D}}\right)$ is quite discrepant w.r.t. the other $T_{\text {eff }}$-values. Concentrating on the most discrepant star, $\mathrm{H}$ Sco, this too high a $T_{\text {eff }}\left(K_{0}, B C_{K}, \theta_{\mathrm{D}}\right)$ value can be either due to too low an $m_{\text {bol }}$ or $\theta_{\mathrm{d}}$ value. The used $K$ magnitude of $0.00 \mathrm{mag}$ is from Price (1968), who quoted this value as being uncertain. We note that an error of +0.4 mag in $K(K=0.4 \mathrm{mag})$, inducing a $0.11 \mathrm{mag}$ decrease in $B C_{K}$ around $4000 \mathrm{~K}$ so that $m_{\mathrm{bol}}=2.99 \mathrm{mag}$, leads to an error in $T_{\text {eff }}$ of $-275 \mathrm{~K}\left(T_{\text {eff }}\left(K_{0}, B C_{K}, \theta_{\mathrm{D}}\right)=3837 \mathrm{~K} ;-367 \mathrm{~K}\right.$ for the increase in $K,+92 \mathrm{~K}$ for in the decrease in $B C_{K}$ ). Simultaneously, this increase in $K$, through a decrease of $V-K$ by $0.4 \mathrm{mag}$, induces an increase of about $75 \mathrm{~K}$ in the $T_{\text {eff }}$ deduced from $T_{\text {eff }}\left((V-K)_{0}\right)$ calibrations. Concerning the bolometric correction $B C_{K}$ we have used the relation found by Bessell et al. (1998). As can be seen from Fig. 20 in Bessell et al. (1998), the $B C_{K}$ values of Bessell \& Wood (1984) are systematically somewhat higher in the region $2.3<V-K<4.5 \mathrm{mag}$, with a maximum difference around $V-K=3$ mag and being almost 0 for $V-K \approx 4$. So, uncertainties in $B C_{K}$ can not explain the observed differences. For $\mathrm{H} \mathrm{Sco}$, only $1 \mathrm{ISO}-\mathrm{SWS}$ observation is available. Inspecting the literature values for the angular diameter for the other cool stars in the sample, we see that $\theta_{\mathrm{d}}$ (ISO) is not systematically higher or lower than the other quoted values. Taking the worst-case scenario, in which the absolute-flux level of the ISO-SWS data is too low by $10 \%$ (a value deduced from the comparison between the different observations of one target, as done in the appendix) and using the same $T_{\text {eff }}$ (ISO) value of $3900 \mathrm{~K}$, the angular diameter of $\mathrm{H}$ Sco would increase from 4.73 mas to 4.96 mas inducing a decrease in $T_{\text {eff }}\left(K_{0}, B C_{K}, \theta_{\mathrm{D}}\right)$ from $4112 \mathrm{~K}$ to $4016 \mathrm{~K}$, still different by $\sim 250 \mathrm{~K}$ from the $T_{\text {eff }}\left((V-K)_{0}\right)$ values. Moreover, as discussed in Sect. 3.10.2, we derive an effective temperature of $T_{\text {eff }}=3900 \mathrm{~K}$ using the Johnson $B, V$, $R$ and $I$ colour measurements of Johnson et al. (1966) and the colour-temperature relation of McWilliam (1990). This value of $3900 \mathrm{~K}$ - being $\sim 150 \mathrm{~K}$ higher than the initial guess value based on $K=0.00 \mathrm{mag}$ - is supported by the slope of the ISOSWS spectrum of H Sco. We may conclude that a least the (high) uncertainty in the $K$ magnitude can explain the larger deviation of $T_{\text {eff }}\left(K_{0}, B C_{K}, \theta_{\mathrm{D}}\right)$ w.r.t. the other $T_{\text {eff }}$ values.

Taking a (more typical) error of -0.1 mag in the $K$ magnitude of $\alpha$ Tuc, would also shift this target in within the $3 \%$ level of correspondence in the lowermost plot of Fig. 14.

Taking all these remarks into account, we may conclude that uncertainties in the $K$ magnitude or in $\theta_{\mathrm{d}}$ may cause $\mathrm{H}$ Sco and $\alpha$ Tuc to deviate from the $3 \%$ level of correspondence of the different obtained $T_{\text {eff-values. Moreover, this high level of }}$ correspondence proves the consistency between $V-K, B C_{K}$, $T_{\text {eff }}$ and $\theta_{\mathrm{d}}$ derived from IR and optical data.

\section{2. ${ }^{12} \mathrm{C} /{ }^{13} \mathrm{C}$ ratio}

Classical models (i.e. those where stellar convective regions are instantly mixed, where no transport of matter occurs in the radiative regions and without rotation at any depth) predict that the carbon isotopic ratio ${ }^{12} \mathrm{C} /{ }^{13} \mathrm{C}$ in low-mass stars $\left(M \lesssim 2 M_{\odot}\right)$ declines from 90 to about 20-30 along the red giant branch (e.g. Iben 1967; Dearborn et al. 1976; Charbonnel et al. 1998). This pattern is, however, not seen for our program stars (see Fig. 15). Observations of CNO elements in a large number of evolved stars (for an overview, see Sneden 1991) permitted already to confirm the qualitative occurrence of the first dredgeup, but quantitative disagreements appeared between the abundance pattern of real low-mass stars and of "model" stars: in a large fraction of giants the observed ${ }^{12} \mathrm{C} /{ }^{13} \mathrm{C}$ and ${ }^{12} \mathrm{C} /{ }^{14} \mathrm{~N}$ ratios appeared to be substantially lower than the predicted postdredge-up ratios. Therefore, different processes involving an extra-mixing process have been proposed to explain the deviations from standard theoretical predictions (for an overview, see Charbonnel 1994; Charbonnel et al. 1998). Observational results of Charbonnel et al. (1998) confirmed that the extramixing process becomes efficient on the RGB only when the low-mass stars reach the so-called luminosity function bump, i.e. the evolutionary point where the hydrogen burning shell crosses the chemical discontinuity created by the convective envelope at its maximum extent, tending to indicate that the nature of this extra-mixing process on the RGB is related to rotation.

Our observational results are summarised in Fig. 15, where we show the observed dependence of the ${ }^{12} \mathrm{C} /{ }^{13} \mathrm{C}$ ratio on $M_{\mathrm{bol}}$. The same conclusions can be drawn as by Charbonnel et al. (1998), namely (i) the least luminous star in our sample, $\xi$ Dra, shows a limit on the ${ }^{12} \mathrm{C} /{ }^{13} \mathrm{C}$ ratio in agreement with standard predictions, and (ii) for stars with $M_{\text {bol }}<0.9$, the observed isotopic ratio drops to values between 7 and 13, well below the standard predicted post-dilution ratio. As quoted by Charbonnel et al. (1998), it is interesting to note that the RGB luminosity function bump of $47 \mathrm{Tuc}$ (with $[\mathrm{Fe} / \mathrm{H}] \simeq-0.65$ ) is at $M_{\text {bol }}=1.05 \pm 0.20$, i.e. precisely where the disagreement between standard predictions and observed ${ }^{12} \mathrm{C} /{ }^{13} \mathrm{C}$ ratios appears. Our observational results, in which for the first time the ${ }^{12} \mathrm{C} /{ }^{13} \mathrm{C}$-ratio of $\delta$ Dra, $\alpha$ Tuc and $\mathrm{H}$ Sco are included, are thus a confirmation of the claims of Charbonnel et al. (1998).

Moreover it has to be noted that for more massive stars $\left(M \geq 1.7-2.3 M_{\odot}\right.$, depending on the metallicity) the 
Table 5. Effective temperatures derived from the $\left(T_{\text {eff }}, V-K\right)$ relationship as given by Ridgway et al. (1980) (RJWW80), di Benedetto (1993, 1998) (diBen9398) and Bessell et al. (1998) (BCP98) are tabulated in Cols. $3-5$ respectively. In Col. 6, $T_{\text {eff }}\left(K_{0}, B C_{K}, \theta_{\mathrm{D}}\right)$ is listed and in the last column the effective temperature deduced from the ISO-SWS spectra is given.

\begin{tabular}{lcccccc}
\hline \hline name & $(V-K)_{0}$ & $\begin{array}{c}T_{\text {eff }}\left((V-K)_{0}\right) \\
\text { RJWW80 }\end{array}$ & $\begin{array}{c}T_{\text {eff }}\left((V-K)_{0}\right) \\
\text { diBen9398 }\end{array}$ & $\begin{array}{c}T_{\text {eff }}\left((V-K)_{0}\right) \\
\text { BCP98 }\end{array}$ & $T_{\text {eff }}\left(K_{0}, B C_{K}, \theta_{\mathrm{D}}\right)$ & $T_{\text {eff }}($ ISO $)$ \\
\hline$\delta$ Dra & 2.26 & 4846 & $4784 \pm 48$ & 4828 & $4858 \pm 157$ & $4820 \pm 140$ \\
$\xi$ Dra & 2.70 & 4437 & $4412 \pm 44$ & 4457 & $4457 \pm 166$ & $4440 \pm 300$ \\
$\alpha$ Boo & 2.88 & 4321 & $4280 \pm 43$ & 4331 & $4226 \pm 139$ & $4320 \pm 140$ \\
$\alpha$ Tuc & 2.91 & 4302 & $4264 \pm 43$ & 4312 & $4052 \pm 133$ & $4300 \pm 140$ \\
$\beta$ UMi & 3.25 & 4101 & $4061 \pm 41$ & 4111 & $3963 \pm 132$ & $4085 \pm 140$ \\
$\gamma$ Dra & 3.54 & 3973 & $3926 \pm 39$ & 3970 & $3978 \pm 134$ & $3960 \pm 140$ \\
$\alpha$ Tau & 3.67 & 3931 & $3874 \pm 39$ & 3915 & $3831 \pm 129$ & $3850 \pm 140$ \\
H Sco & 4.08 & 3793 & $3688 \pm 98$ & 3764 & $4112 \pm 139$ & $3900 \pm 140$ \\
$\beta$ And & 3.82 & 3845 & $3722 \pm 95$ & 3856 & $3790 \pm 127$ & $3880 \pm 140$ \\
$\alpha$ Cet & 4.07 & 3796 & $3689 \pm 98$ & 3768 & $3679 \pm 124$ & $3740 \pm 140$ \\
$\beta$ Peg & 4.61 & 3648 & $3622 \pm 102$ & 3615 & $3554 \pm 147$ & $(3600)$ \\
\hline
\end{tabular}

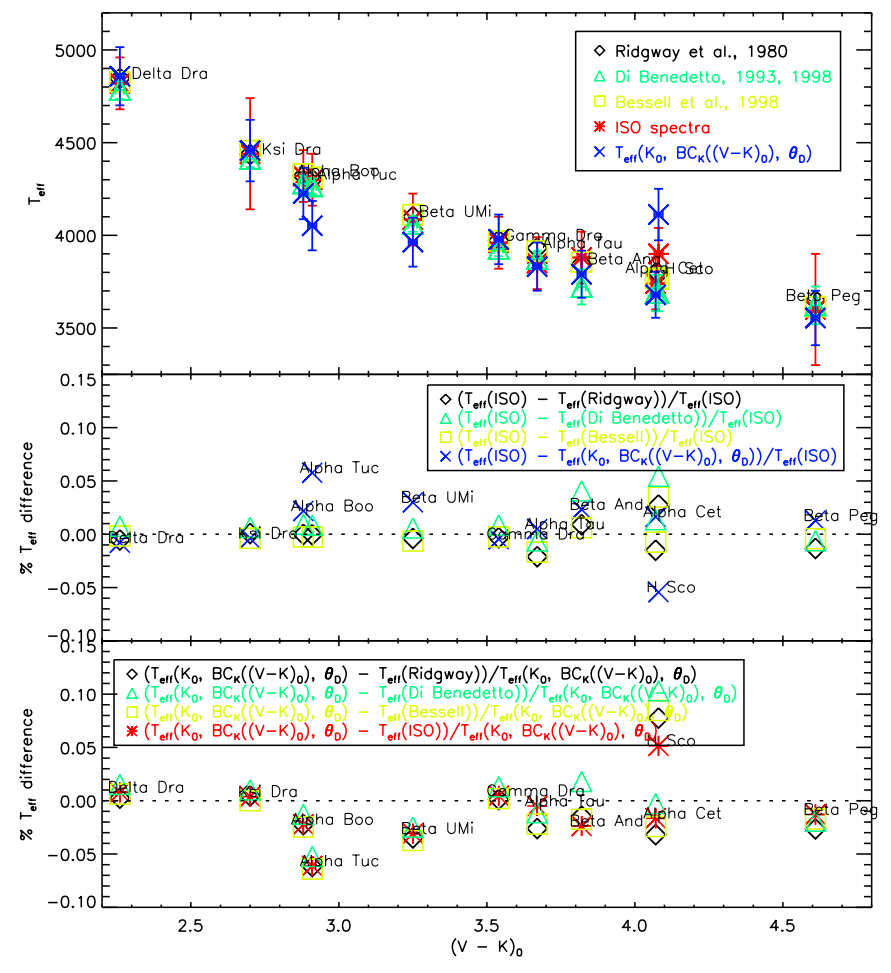

Fig. 14. Comparison between $T_{\text {eff }}(V-K)$ derived from the relationship given by Ridgway et al. (1980), di Benedetto (1993), di Benedetto (1998) or Bessell et al. (1998), $T_{\text {eff }}\left(K_{0}, B C_{K}, \pi\right)$ and the effective temperature deduced from the ISO-SWS spectra. A coloured version of this plot is available in the appendix as Fig. C.1.

evolutionary scenario is different: the hydrogen-burning shell never reaches the region of constant molecular weight that has been homogenised by the convective envelope during the first dredge-up. Under these conditions, the extra-mixing process does not occur in these stars and their abundance pattern should resemble the pattern predicted by standard stellar theory. Taking into account the (large) error bars on the derived $M_{\mathrm{g}}$

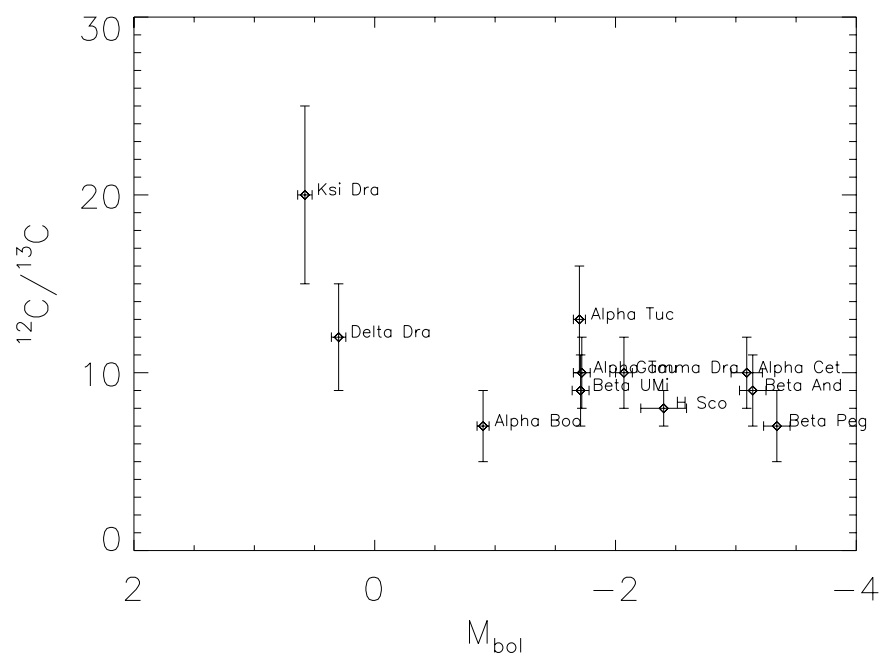

Fig. 15. The ratio of ${ }^{12} \mathrm{C} /{ }^{13} \mathrm{C}$ is plotted against the bolometric magnitude of the program stars. The absolute magnitudes are tabulated in Table 2. This figure should be compared with Fig. 1 in Charbonnel et al. (1998).

values or on the assumed $M_{\mathrm{hr}}$ values, and assuming that the cool giants in our sample are still on the RGB - see also the discussion in the previous section - and that no other extramixing process occurs, the low values of the ${ }^{12} \mathrm{C} /{ }^{13} \mathrm{C}$ ratios are indicative for low-mass stars $\left(M \lesssim 2 M_{\odot}\right)$ in which this extramixing process occurs.

\section{Conclusions}

In this paper, the atmospheric parameters of 11 giants have been determined by using the method described in Papers I and II. A confrontation with other published stellar parameters, other ISO-SWS data and the synthetic spectra always showed very "positive" results both for the theoretical modelling and for the calibration of the ISO-SWS detectors. The very small 
discrepancies still remaining in band 1 are at the $1-2 \%$ level for the giants, proving not only that the calibration of the (highflux) sources in this band is better than $\sim 2 \%$, but also that the description of cool-star atmospheres and molecular linelists is already quite accurate at the ISO-SWS spectral resolution $(R \sim 1000)$.

In the discussion of the deduced parameters, we demonstrated that $T_{\text {eff }}$ (ISO) is consistent with other $T_{\text {eff }}\left((V-K)_{0}\right)$ or $T_{\text {eff }}\left(K_{0}, B C_{K}, \theta_{\mathrm{d}}\right.$ (ISO)) values in within the few percent level, proving once more that the used MARCs-models to derive the quantities from the ISO-SWS spectra are very trustworthy and that the flux calibration of ISO-SWS is indeed reassuring.

The obtained ${ }^{12} \mathrm{C} /{ }^{13} \mathrm{C}$ ratios are compatible with other sources (e.g. Charbonnel 1995).

Like already pointed out in Paper II, these synthetic spectra will now be used for the - latest - OLP10 calibration of ISO-SWS. Also other consortia constructing instruments for ground-based telescopes - i.e. MIDI (=the Mid-Infrared Interferometric instrument for the VLTI with first observations foreseen in 2001) and new satellites - i.e. SIRTF, the Space InfraRed Telescope Facility, with launch foreseen in December 2001, and Herschel, a far-infrared submillimeter telescopes with launch foreseen in 2007 - will use this type of study and/or these SEDs for determining the required sensitivity of their instruments. A confrontation with existing SEDs, like the ones of M. Cohen, is therefore planned in a forthcoming paper of this series, in which also the new atomic linelist constructed by Sauval (Sauval 2002) will be implemented.

Acknowledgements. LD acknowledges support from the Science Foundation of Flanders. This research has made use of the SIMBAD database, operated at CDS, Strasbourg, France and of the VALD database, operated at Vienna, Austria. It is a pleasure to thank the referees, J. Hron and F. Kupka, for their careful reading of the manuscript and for their valuable suggestions.

\section{References}

Abia, C., \& Wallerstein, G. 1998, MNRAS, 293, 89

Altas, L. 1987, Ap\&SS, 134, 85

Anders, E., \& Grevesse, N. 1989, Geochim. Cosmochim. Acta, 53, 197

Aoki, W., \& Tsuji, T. 1997, A\&A, 328, 175

Aringer, B., Höfner, S., Wiedemann, G., et al. 1999, A\&A, 342, 799

Ayres, T. R., Simon, T., \& Linsky, J. L. 1982, ApJ, 263, 791

Barnes, T. G., \& Evans, D. S. 1976, MNRAS, 174, 489

Barnes, T. G., Evans, D. S., \& Parsons, S. B. 1976, MNRAS, 174, 503

Basri, G. S., \& Linsky, J. L. 1979, ApJ, 234, 1023

Bell, R. A., Edvardsson, B., \& Gustafsson, B. 1985, MNRAS, 212, 497

Bell, R. A., \& Gustafsson, B. 1989, MNRAS, 236, 653

Bessell, M. S., Castelli, F., \& Plez, B. 1998, A\&A, 333, 231

Bessell, M. S. \& Wood, P. R. 1984, PASP, 96, 247

Blackwell, D. E., Lynas-Gray, A. E., \& Petford, A. D. 1991, A\&A, 245,567

Blackwell, D. E., Petford, A. D., Haddock, D. J., Arribas, S., \& Selby, M. J. 1990, A\&A, 232, 396
Blackwell, D. E., \& Lynas-Gray, A. E. 1998, A\&AS, 129, 505

Blackwell, D. E., Petford, A. D., \& Shallis, M. J. 1980, A\&A, 82, 249

Blackwell, D. E., \& Shallis, M. J. 1977, MNRAS, 180, 177

Böhm-Vitense, E. 1981, ARA\&A, 19, 295

Bonnell, J., \& Bell, R. A. 1982, MNRAS, 201, 253

Bonnell, J. T., \& Bell, R. A. 1993, MNRAS, 264, 319

Brown, J. A., Sneden, C., Lambert, D. L., \& Dutchover, E. J. 1989, ApJS, 71, 293

Burnashev, V. I. 1983, Izvestiya Ordena Trudovogo Krasnogo Znameni Krymskoj Astrofizicheskoj Observatorii, 67, 13

Cami, J., Justtanont, K., de Jong, T., et al. 1997, in The first ISO workshop on Analytical Spectroscopy, ed. A. M. Heras, K. Leech, N. R. Trams, \& M. Perry, ESA SP-419, 159

Cayrel de Strobel, G., Hauck, B., Francois, P., et al. 1992, A\&AS, 95, 273

Charbonnel, C. 1994, A\&A, 282, 811

Charbonnel, C. 1995, ApJ, 453, L41

Charbonnel, C., Brown, J. A., \& Wallerstein, G. 1998, A\&A, 332, 204

Clegg, R. E. S., Lambert, D. L., \& Bell, R. A. 1979, ApJ, 234, 188

Cohen, M., Walker, R. G., \& Witteborn, F. C. 1992, AJ, 104, 2030

Cohen, M., Witteborn, F. C., Carbon, D. F., et al. 1996, AJ, 112, 2274

Cornide, M., Fernandez-Figueroa, M. J., de Castro, E., Armentia, J. A., \& Reglero, V. 1992, AJ, 103, 1374

Currie, D. G., Knapp, S. L., \& Liewer, K. M. 1974, ApJ, 187, 131

Davis, J. 1998, in Fundamental Stellar Properties, IAU Symp., 189, 31 de Jong, T. 1999, in Abstracts of the 2nd Austrian ISO Workshop, Atmospheres of M, S and C Giants, ed. J. Hron, \& S. Höfner, 53

Dearborn, D. S. P., Eggleton, P. P., \& Schramm, D. N. 1976, ApJ, 203, 455

Dearborn, D. S. P., Lambert, D. L., \& Tomkin, J. 1975, ApJ, 200, 675

Decin, L. 2000, Ph.D. Thesis, University of Leuven

Decin, L., Cohen, M., Eriksson, K., et al. 1997, in The first ISO workshop on Analytical Spectroscopy, ed. A. M. Heras, K. Leech, N. R. Trams, \& M. Perry, ESA SP-419, 185

Decin, L., Vandenbussche, B., Waelkens, C., et al. 2003, A\&A, 400, 679 (Paper II)

Decin, L., Waelkens, C., Eriksson, K., et al. 2000, A\&A, 364, 137

di Benedetto, G. P. 1993, A\&A, 270, 315

di Benedetto, G. P. 1998, A\&A, 339, 858

di Benedetto, G. P., \& Rabbia, Y. 1987, A\&A, 188, 114

Drira, I., Hure, J. M., Spielfiedel, A., Feautrier, N., \& Roueff, E. 1997, A\&A, 319, 720

Dyck, H. M., Lockwood, G. W., \& Capps, R. W. 1974, ApJ, 189, 89

Dyck, H. M., van Belle, G. T., \& Thompson, R. R. 1998, AJ, 116, 981

Edvardsson, B. 1988, A\&A, 190, 148

Eggen, O. J. 1973, PASP, 85, 379

El Eid, M. F. 1994, A\&A, 285, 915

Engelke, C. W. 1992, AJ, 104, 1248

Fagotto, F., Bressan, A., Bertelli, G., \& Chiosi, C. 1994, A\&AS, 105, 29

Faucherre, M., Bonneau, D., Koechlin, L., \& Vakili, F. 1983, A\&A, 120,263

Fernández-Villacañas, J. L., Rego, M., \& Cornide, M. 1990, AJ, 99, 1961

Flynn, C., \& Mermilliod, J. 1991, A\&A, 250, 400

Frisk, U., Nordh, H. L., Olofsson, S. G., Bell, R. A., \& Gustafsson, B. 1982, MNRAS, 199, 471

Gadun, A. S. 1994, Astronomische Nachrichten, 315, 413

Gezari, D. Y., Labeyrie, A., \& Stachnik, R. V. 1972, ApJ, 173, L1

Gezari, D. Y., Pitts, P. S., \& Schmitz, M. 1999, VizieR Online Data Catalog, 2225, 0

Girardi, L., Bressan, A., Bertelli, G., \& Chiosi, C. 2000, A\&AS, 141, 371 
Glass, I. S. 1974, Monthly Notes of the Astronomical Society of South Africa, 33, 53

Glebocki, R., \& Stawikowski, A. 1988, A\&A, 189, 199

Gliese, W. 1969, Veröffentlichungen des Astronomishen RechenInstituts Heidelberg, 22, 1

Gratton, L., Gaudenzi, S., Rossi, C., \& Gratton, R. G. 1982, MNRAS, 201, 807

Gratton, R. G. 1983, MNRAS, 202, 231

Gratton, R. G. 1985, A\&A, 148, 105

Gray, D. F. 1992, The observation and analysis of stellar photospheres, Cambridge Astrophys. Ser., 2nd ed. (Cambridge: Cambridge University Press)

Griffin, R. 1968, A photometric atlas of the spectrum of Arcturus, $\lambda \lambda 3600-8825 \AA$ (Cambridge: Cambridge Philosophical Society)

Gustafsson, B., \& Bell, R. A. 1979, A\&A, 74, 313

Gustafsson, B., Bell, R. A., Eriksson, K., \& Nordlund, Å. 1975, A\&A, 42,407

Gustafsson, B., Kjærgaard, P., \& Andersen, S. 1974, A\&A, 34, 99

Hammersley, P. L., Jourdain de Muizon, M., Kessler, M. F., et al. 1998, A\&AS, 128, 207

Harris, M. J., \& Lambert, D. L. 1984, ApJ, 285, 674

Harris, M. J., Lambert, D. L., \& Smith, V. V. 1988, ApJ, 325, 768

Hinkle, K. H., Hall, D. N. B., \& Ridgway, S. T. 1982, ApJ, 252, 697

Hoffleit, D., \& Jaschek, C. 1982, The Bright Star Catalogue, The Bright Star Catalogue (New Haven: Yale University Observatory), 4th edition

Höfner, S., Loidl, R., Aringer, B., \& Jørgensen, U. 2002, in Radial and Nonradial Pulsations as Probes of Stellar Physics, ed. C. Aerts, T. Bedding, \& J. Christensen-Dalsgaard, ASP Conf. Ser., in press (San Francisco: ASP)

Hutter, D. J., Johnston, K. J., Mozurkewich, D., et al. 1989, ApJ, 340, 1103

Johnson, H. L. 1966, ARA\&A, 4, 193

Judge, P. G. 1986, MNRAS, 221, 119

Iben, I. J. 1967, ApJ, 147, 624

Jenkins, L. F. 1952, General catalogue of trigonometric stellar parallaxes (New Haven: Yale University Observatory)

Jenkins, L. F. 1963, in General Cat. Trigo. Parallaxes (1963), 0

Johnson, H. L., Iriarte, B., Mitchell, R. I., \& Wisniewskj, W. Z. 1966, Communications of the Lunar and Planetary Laboratory, 4, 99

Judge, P. G., \& Stencel, R. E. 1991, ApJ, 371, 357

Justtanont, K., Feuchtgruber, H., de Jong, T., et al. 1998, A\&A, 330, L17

Kjærgaard, P., Gustafsson, B., Walker, G. A. H., \& Hultqvist, L. 1982, A\&A, 115, 145

Koechlin, L., \& Rabbia, Y. 1985, A\&A, 153, 91

Kovács, N. 1983, A\&A, 120, 21

Kyröläinen, J., Tuominen, I., Vilhu, O., \& Virtanen, H. 1986, A\&AS, 65,11

Lambert, D. L. 1976, Mémoires of the Société Royale des Sciences de Liege, 9, 405

Lambert, D. L., Dominy, J. F., \& Sivertsen, S. 1980, ApJ, 235, 114

Lambert, D. L., McWilliam, A., \& Smith, V. V. 1987, Ap\&SS, 133, 369

Lambert, D. L., \& Ries, L. M. 1981, ApJ, 248, 228

Langhoff, S. R., \& Bauschlicher, C. W. J. 1993, Chem. Phys. Lett., 211, 305

Lazaro, C., Lynas-Gray, A. E., Clegg, R. E. S., Mountain, C. M., \& Zadrozny, A. 1991, MNRAS, 249, 62

Lee, T. A. 1970, ApJ, 162, 217

Leggett, S. K., Mountain, C. M., Selby, M. J., et al. 1986, A\&A, 159, 217

Lejeune, T., \& Schaerer, D. 2001, A\&A, 366, 538
Linsky, J. L., \& Ayres, T. R. 1978, ApJ, 220, 619

Linsky, J. L., McClintock, W., Robertson, R. M., \& Worden, S. P. 1979, ApJS, 41, 47

Luck, R. E., \& Challener, S. L. 1995, AJ, 110, 2968

Mäckle, R., Griffin, R., Griffin, R., \& Holweger, H. 1975, A\&AS, 19, 303

Manduca, A. 1979, A\&AS, 36, 411

Manduca, A., Bell, R. A., \& Gustafsson, B. 1981, ApJ, 243, 883

Mathis, J. S. 1990, ARA\&A, 28, 37

McWilliam, A. 1990, ApJS, 74, 1075

Mendoza, E. E. 1969, Boletin de los Observatorios Tonantzintla y Tacubaya, 5, 57

Mermilliod, J.-C. 1986, Compilation of Eggen's UBV data, transformed to $U B V$ (SIMBAD)

Moon, T. 1985, Ap\&SS, 117, 261

Morossi, C., Franchini, M., Malagnini, M. L., Kurucz, R. L., \& Buser, R. 1993, A\&A, 277, 173

Mozurkewich, D., Johnston, K. J., Simon, R. S., et al. 1991, AJ, 101, 2207

Napiwotzki, R., Schönberner, D., \& Wenske, V. 1993, A\&A, 268, 653

Nicolet, B. 1978, A\&AS, 34, 1

Oinas, V. 1977, A\&A, 61, 17

Pasquini, L., \& Brocato, E. 1992, A\&A, 266, 340

Pasquini, L., Brocato, E., \& Pallavicini, R. 1990, A\&A, 234, 277

Perrin, G., Coude Du Foresto, V., Ridgway, S. T., et al. 1998, A\&A, 331,619

Pilachowski, C., Sneden, C., Hinkle, K., \& Joyce, R. 1997, AJ, 114, 819

Peterson, R. C., Dalle Ore, C. M., \& Kurucz, R. L. 1993, ApJ, 404, 333

Plez, B., Brett, J. M., \& Nordlund, Å. 1992, A\&A, 256, 551

Plez, B., Smith, V. V., \& Lambert, D. L. 1993, ApJ, 418, 812

Price, S. D. 1968, AJ, 73, 431

Quirrenbach, A., Mozurkewich, D., Armstrong, J. T., Buscher, D. F., \& Hummel, C. A. 1993, ApJ, 406, 215

Quirrenbach, A., Mozurkewich, D., Buscher, D. F., Hummel, C. A., \& Armstrong, J. T. 1996, A\&A, 312, 160

Ridgway, S. T., Joyce, R. R., White, N. M., \& Wing, R. F. 1980, ApJ, 235,126

Ries, L. M. 1981, Ph.D. Thesis, Texas Univ., Arlington

Robinson, R. D., Carpenter, K. G., \& Brown, A. 1998, ApJ, 503, 396

Rodgers, B., \& Glassgold, A. E. 1991, ApJ, 382, 606

Ryde, N., Eriksson, K., \& Gustafsson, B. 1999, A\&A, 341, 579

Sanner, F. 1976, ApJS, 32, 115

Sauval, A. J. 2002, Data file of IR solar atomic lines, unpublished results

Scalo, J. M. 1976, ApJ, 206, 474

Scalo, J. M., \& Miller, G. E. 1978, ApJ, 225, 523

Scargle, J. D., \& Strecker, D. W. 1979, ApJ, 228, 838

Smith, G., Edvardsson, B., \& Frisk, U. 1986, A\&A, 165, 126

Smith, V. V., \& Lambert, D. L. 1985, ApJ, 294, 326

Smith, V. V., \& Lambert, D. L. 1990, ApJS, 72, 387

Sneden, C. 1974, Ph.D. Thesis, University of Texas

Sneden, C. 1991, in IAU Symp. 145, Evolution of Stars: The Photospheric Abundance Connection, ed. G. Michaud, \& A. V. Tutukov, 235

Stencel, R. E., Linsky, J. L., Mullan, D. J., Basri, G. S., \& Worden, S. P. 1980, ApJS, 44, 383

Taylor, B. J. 1999, A\&AS, 134, 523

Tielens, A. G. G. M. 1983, ApJ, 271, 702

Tipping, R. H., \& Chackerian, C. J. 1981, J. Mol. Spectr., 88, 352

Tomkin, J., Lambert, D. L., \& Luck, R. E. 1975, ApJ, 199, 436

Tomkin, J., Luck, R. E., \& Lambert, D. L. 1976, ApJ, 210, 694 
Tsuji, T. 1981, A\&A, 99, 48

Tsuji, T. 1986, A\&A, 156, 8

Tsuji, T. 1991, A\&A, 245, 203

Tsuji, T., Ohnaka, K., Aoki, W., \& Yamamura, I. 1997, A\&A, 320, L1

Tsuji, T., Ohnaka, K., Hinkle, K. H., \& Ridgway, S. T. 1994, A\&A, 289, 469

van Paradijs, J. 1973, A\&A, 23, 369

van Paradijs, J., \& Kester, D. 1976, A\&A, 53, 1

van Paradijs, J., \& Meurs, E. J. A. 1974, A\&A, 35, 225

Volk, K., \& Cohen, M. 1989, AJ, 98, 1918

Wallace, L., Bernath, P., Livingston, W., et al. 1995, Science, 268, 1155
Wamsteker, W. 1981, A\&A, 97, 329

Wilson, O. C. 1976, ApJ, 205, 823

Woitke, P., Helling, C., Winters, J. M., \& Jeong, K. S. 1999, A\&A, 348, L17

Worthey, G. 1994, ApJS, 95, 107

Worthey, G., Faber, S. M., Gonzalez, J. J., \& Burstein, D. 1994, ApJS, 94, 687

Yamamura, I., de Jong, T., Onaka, T., Cami, J., \& Waters, L. B. F. M. 1999, A\&A, 341, L9 